\title{
2004 MRS Spring Meeting/UMRS-ICEM 2004 Brings Together Emerging and Established Fields in Materials Research
}

The 2004 Materials Research Society Spring Meeting, in conjunction with the International Union of Materials Research Societies' 9th International Conference on Electronic Materials (IUMRS-ICEM 2004), brought together news on rapidly breaking areas as well as overviews of established fields in materials research. Held April 12-16 in San Francisco, California, the Meeting showed a notable increase in the ways nanomaterials feed into biomaterials. Meeting chairs Israel J. Baumvol (UFRGS, Instituto de Fisica), James J. De Yoreo (Lawrence Livermore National Laboratory), Siegfried Mantl (Forschungszentrum Jülich), and Thomas X. Neenan (Genzyme Corporation) divided the 28 technical symposia into four clusters: Electronics, Spintronics, and Photonics (Symposia A-L); Nano- and Microstructured Materials (M-S); Molecular, Biological, and Hybrid Materials (T-AA); and General (Symposium $X$ on Frontiers of Materials Research, BB on Education, and CC on Waste Management), all held in the new venue of the Moscone West Convention Center. The Meeting included five days of technical sessions and talks, poster sessions, the awards and plenary session, tutorials, special events, exhibit, and other activities, with more than 2000 papers presented in oral and poster sessions to over 2700 attendees.

\section{Technical Talks}

Are flexible displays ready for "prime time"? Not quite, said K. Allen (iSuppli/ Standford Resources), who gave the first talk in Symposium I. However, progress on all fronts looks promising, she said, with continued development likely to be able to handle foreseeable obstacles. Some challenges remain across the board, from finding a substrate that repeatedly flexes yet retains dimensional stability through temperature changes, to finding a way to get a pattern onto a flexible substrate. While applications, such as electrophoretic dynamic signage, are emerging and are likely to grow through this decade, Allen said that there is no "killer app," an application that will drive this technology to large-scale commercialization because of its unique need for this solution. While early images touted the idea of roll-to-roll processing, batch processing seems more suited to many likely applications, she said.

Also within the realm of flexible electronics, the topic of Symposium I, a big issue in small-molecule LEDs, particularly flexible ones, is the concern of how to block moisture. With an image of a crumbling wall of bricks trying to hold out a sea of water, P.E. Burrows (PNNL) described just how permeable to water the organic films are. He described a multilayer system that works, although at first glance its success seems unlikely. The alternating layers of polymer and ceramic serve to decouple defects from previous layers (with the planarizing polymer) and to block the flow of moisture (due to the thin aluminum oxide ceramic layer). While equilibrium calculations show that the moisture cannot be kept out with such a barrier, the reality is that the films do work. This is due, he said, to the more realistic nonequilibrium kinetics, which demonstrates a lag time that increases with more layers, buying time on the order of years before permeation causes destruction. Burrows also introduced a technique for rapid, continuous production of OLEDs, using several external syringe pumps that feed a liquid solution into a box, where the material is vaporized and the right mix and temperature are maintained and fed to produce the OLED films.

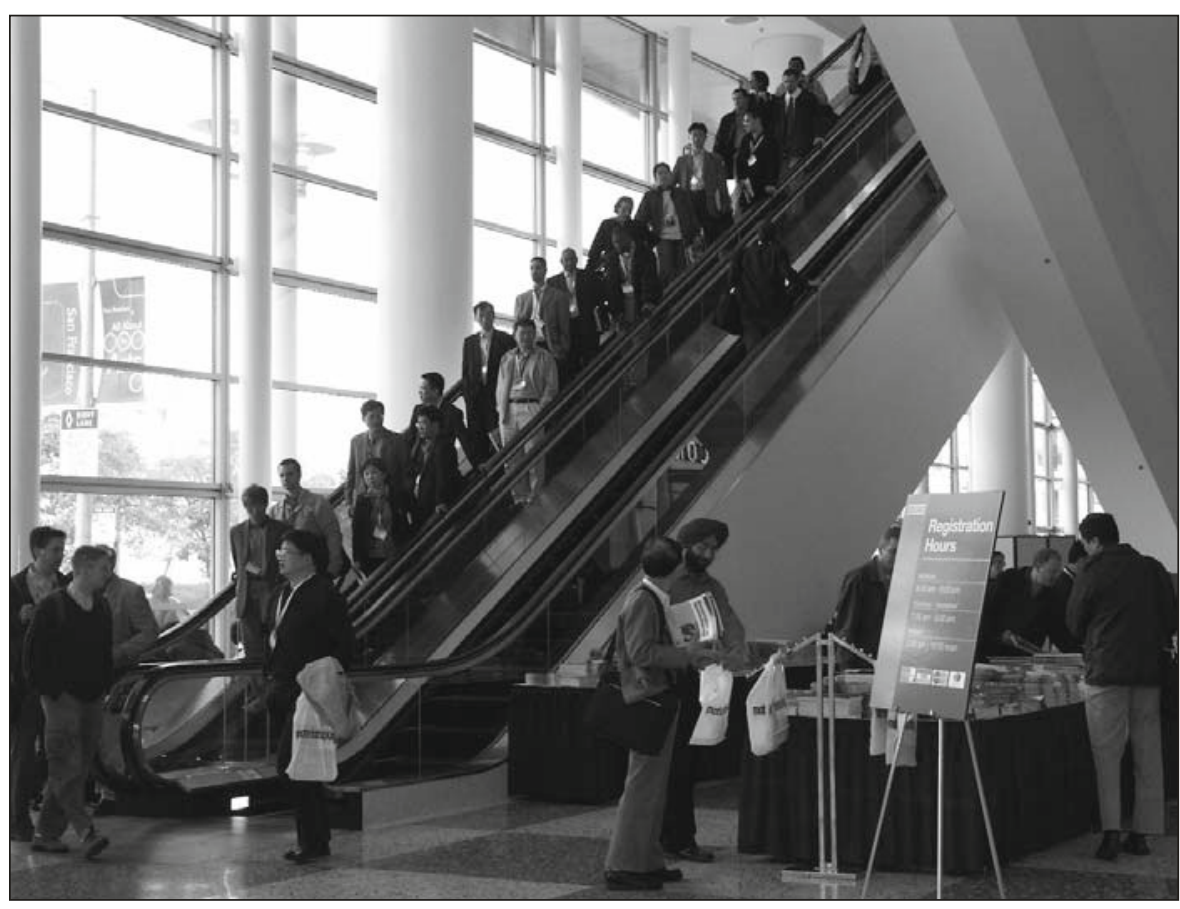

The Moscone West Convention Center served as the new venue for the MRS Spring Meeting. 
In back-to-back presentations for a joint session between Symposia $\mathrm{W}$ and $\mathrm{O}$ and in Symposium U, Harvard professor G.M. Whitesides introduced some approaches to nanofabrication. In his first talk, Whitesides said that a planar microfabrication approach is more practical than the "beads-in-a-string" concept observed in nature and typically used in biomimetic approaches to self-assembly. As an example, a crimped sheet could be stretched flat to facilitate the printing of a circuit, and then released, thereby forming a 3D circuit. In another example, cartographical techniques were employed to shape virtually flat pieces onto which circuits were printed and that could later be assembled into spherical circuits. Whitesides also showed how, using common 2D substrates, one-, two-, and three-dimensional structures could be constructed using volume-constrained self-assembly.

In his second talk, Whitesides reviewed several unconventional approaches to nanofabrication advanced in his laboratory. Electron-beam writing was shown to form topographically patterned films with an ultimate resolution that is about an order of magnitude better than currently achieved by atomic force microscopy. Examples of electric microcontact printing,

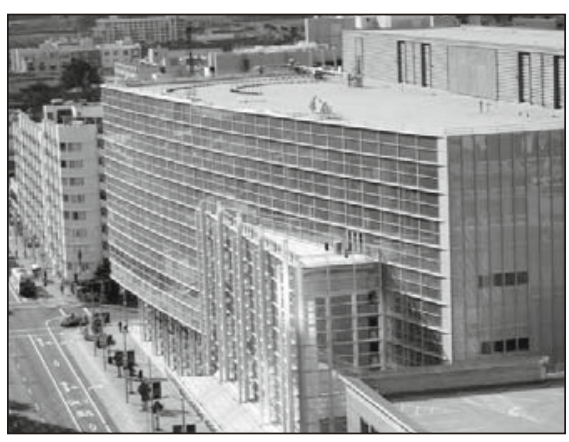

The 2004 Materials Research Society Spring Meeting, in conjunction with the International Union of Materials Research Societies' 9th International Conference on Electronic Materials (IUMRS-ICEM 2004), was held at the Moscone West in San Francisco, Calif., on April 12-16.

edge lithography, and templated synthesis were also shown.

Several more patterning techniques were described in Symposium U on printing of materials. T. Lindner (HP) said that in printing a variety of materials, thermal inkjet technology is by no means limited to aqueous fluids. It can be extended to nonaqueous fluids and enables precision metering and patterning, thus rendering it a very versatile technology. In a historical overview on the development of the technology over the past two decades, Lindner said that thermal inkjet technology has followed Moore's law with doubling of performance every 18 months. The printing of passive/active electronic devices by this technology has passed feasibility testing. Because of the complexity of this technique, Lindner said that the ability to print new non-ink materials would require new designs and architectures. Still, he said, the capability to build circuits is an emerging reality.

This presentation was followed by a talk by G. Jabbour (now at ASU) on combinatorial inkjet printing for organic optoelectronics. Organic functional materials are seeing increasing applications in various devices and displays. Inkjet printing represents a way to easily and inexpensively print these materials on a variety of substrates; however, device optimization is a prerequisite for improving performance and ultimately for lowering cost. Combinatorial methods in conjunction with inkjet printing could meet these needs. Jabbour demonstrated anode pat-

\section{ACRONYM KEY}

2D: two-dimensional

3D: three-dimensional

AFOSR: Air Force Office of Scientific Research

ANL: Argonne National Laboratory

ARL: U.S. Army Research Laboratory

ASU: Arizona State University

BEOL: back-end-of-line

BST: barium strontium titanate

CalPoly: California Polytechnic State University

Caltech: California Institute of Technology

CCD: charge-coupled device

CIMIT: Center for Integration of Medicine and Innovative Technology

CMOS: complementary metal oxide semiconductor

CMP: chemical-mechanical polishing

CMU: Carnegie Mellon University

CWRU: Case Western Reserve University

DARPA: Defense Advanced Research

Projects Agency

EPFL: Ecole Polytechnique Fédérale de Lausanne

FeFET: ferroelectric field-effect transistor

FRAM: ferroelectric random-access memory

Georgia Tech: Georgia Institute of Technology

HP: Hewlett-Packard

IC: integrated circuit

ITO: indium tin oxide

ITRS: International Technology Roadmap for Semiconductors
JHU: Johns Hopkins University

JNCASR: Jawaharlal Nehru Centre for

Advanced Scientific Research

LANL: Los Alamos National Laboratory

LED: light-emitting diode

LEP: light-emitting polymer

LLNL: Lawrence Livermore National Laboratory

MBE: molecular-beam epitaxy

MD: molecular dynamics

MEMS: microelectromechanical systems

MIT: Massachusetts Institute of Technology

MODFET: modulation-doped field-effect transistor

MOSFET: metal oxide semiconductor field-

effect transistor

MPI: Max Planck Institute

NASA: National Aeronautics and Space

Administration

NCSU: North Carolina State University

NEMS: nanoelectromechanical systems

NIBIB: National Institute of Biomedical

Imaging and Bioengineering

NIH: National Institutes of Health

NIST: National Institute of Standards and Technology

NREL: National Renewable Energy Laboratory

NRL: Naval Research Laboratory

NSF: National Science Foundation

OLED: organic light-emitting diode
ONR: Office of Naval Research

ORNL: Oak Ridge National Laboratory

OTFT: organic thin-film transistor

PARC: Palo Alto Research Center

PECVD: plasma-enhanced chemical vapor deposition

PLED: polymer light-emitting device

PNNL: Pacific Northwest National Laboratory

PV: photovoltaic

PZT: lead zirconium titanate

R\&D: research and development

RF: radio frequency

RTP: rapid thermal processing

SNL: Sandia National Laboratories

SOI: silicon-on-insulator

STI: shallow trench isolation

SUNY: State University of New York

TCO: transparent conducting oxide

TEM: transmission electron microscopy

TFT: thin-film transistor

TI: Texas Instruments

UC: University of California

UCLA: University of California-Los

Angeles

UCSB: University of California-Santa Barbara

UCSD: University of California-San Diego

UV-VIS-IR: ultraviolet-visible-infrared

XRD: $x$-ray diffraction 
terning of OLEDs by inkjet printing. The substrate was designed on a computer and printed using various combinations on the same page. The sheet-resistance changes were then measured for the different combinations. The technique is very promising, he said, although significant work still remains.

In Symposium D on high- $\kappa$ insulators and ferroelectrics, O. Auciello (ANL) introduced a $\mathrm{TiAlO}_{x}$ alloy as an alternative gate oxide material for CMOS devices. The 3-20 nm TiAl films were grown on $n$-Si and subjected to in situ oxidation using molecular and atomic oxygen. The $\mathrm{TiAlO}_{x}$ thus formed was amorphous and the layer formed using atomic oxygen annealing showed a leakage current 150 times lower than the one using molecular oxygen. $\mathrm{TiAlO}_{x}$ layers with equivalent oxygen thickness $<1 \mathrm{~nm}$ were achieved on Si using room-temperature oxidation with atomic oxygen. With bandgaps of up to $4 \mathrm{eV}$, Auciello stressed that amorphous $\mathrm{TiAlO}_{x}$ is a candidate for the next generation of high- $\kappa$ dielectrics.

As the push continues for more featurerich, multimode, multiband, wireless communications, so does the need for integration of advanced materials that can increase functionality and decrease the footprint. In Symposium D, S.R. Gilbert (Agilent) reviewed recent advances in the use of perovskites for front-end RF applications and examined the processing and materials needs to integrate these materials. Materials such as PZT and BST are of interest because of their large zero-bias permittivity, which means their dielectric constants can be tuned. Looking at PZT for bulk acoustic resonator devices, Gilbert described how the area can be reduced 40-fold compared to using AlN due to the high electromechanical coupling coefficient, which means a high dielectric constant that can be obtained. Thin-film BST for varactors also can give a reduced area due to its large permittivity. BST, furthermore, has the advantage of not needing a hermetic package.

A.K. Agarwal (Cree) gave an overview of $\mathrm{SiC}$ power devices in Symposium J, describing the future as bright, but with problems yet to solve. While Schottky diodes have already been commercialized, to make use of SiC's performance at high temperatures, the materials interfacing the $\mathrm{SiC}$ need to be replaced or modified. Some remaining issues include improvement on the reliability of the gate oxide, activation of the $p$-well without causing surface roughness, and reduction in fixed oxide charge.

In the cluster of symposia on nano- and microstructured materials, $H$. van
Swygenhoven (Paul Scherrer Institute, Switzerland) in Symposium P reported recent progress in the field of atomistic

simulation of deformation of nanocrystalline materials. An important issue in recent years was the role of partial disloca-

\section{IUMRS Sōmiya Award Honors Collaborative Research of Rao and Cheetham}

During the Plenary/Awards ceremony, C.N.R. Rao (Jawaharlal Nehru Centre for Advanced Scientific Research, Bangalore, India) and Anthony Cheetham (UCSB) received the Sōmiya Award from the IUMRS in recognition of their collaboration on the synthesis and characterization of novel materials. The Sōmiya Award, named in honor of Shigeyuki Sōmiya, Emeritus Professor of the Tokyo Institute of Technology, and later Dean at Teikyo University of Science and Engineering, recognizes research on real materials conducted by a research team whose members are drawn from at least two continents.

In a special presentation at the 2004 MRS Spring Meeting/IUMRS-ICEM 2004 Meeting, Rao and Cheetham each delivered a talk on research they are conducting with their respective research teams. Rao, along with his numerous collaborators and students, has done significant work on forming nanomaterials through soft chemistry. Among the various projects he described, Rao explained their work with solvothermal synthesis, using capping agents, of nanocrystals of materials such as $\mathrm{ZnO}, \mathrm{CdO}, \mathrm{CdS}$, and GaN. Using this technique, for example, they have been able to obtain excellent quality single-crystal GaN nanocrystals. Rao also described his work with obtaining 2D organization of metal nanocrystals, the use of dip-pen lithography using an atomic force microscope tip to form patterns using nanoparticles, and his research relating to nanotubes, including the use of organometallic precursors, one-step synthesis of carbon nanotubes, and synthesis of junction nanotubes in a reproducible fashion.

Cheetham followed with a discussion of work by his group on direct observations of the structure of $\mathrm{Au}$ nanoparticles using powder neutron diffraction. Nanoparticles give very poor diffraction patterns with x-rays or neutrons. Thus, total scattering neutron diffraction was performed using monodispersed $\mathrm{Au}$ nanoparticles that were carefully synthesized forming capped Au nanoparticles so as to reduce incoherent neutron scattering. Fourier transforms of the neutron data yielded pair-distributed functions, which reveals vectors between all atoms in a particle. For a nanoparticle, the maximum length of the vector is determined by its size. The data on pair-distributed functions can be fitted by modeling nanoparticle structure and refined. This yields lattice parameters, temperature factors, and other data. Cheetham said that this method can be extended to other systems, including the study of core-shell structures, nanowires, and carbon nanotubes, and that it is also possible to perform the experiment with short wavelength synchrotron x-rays.

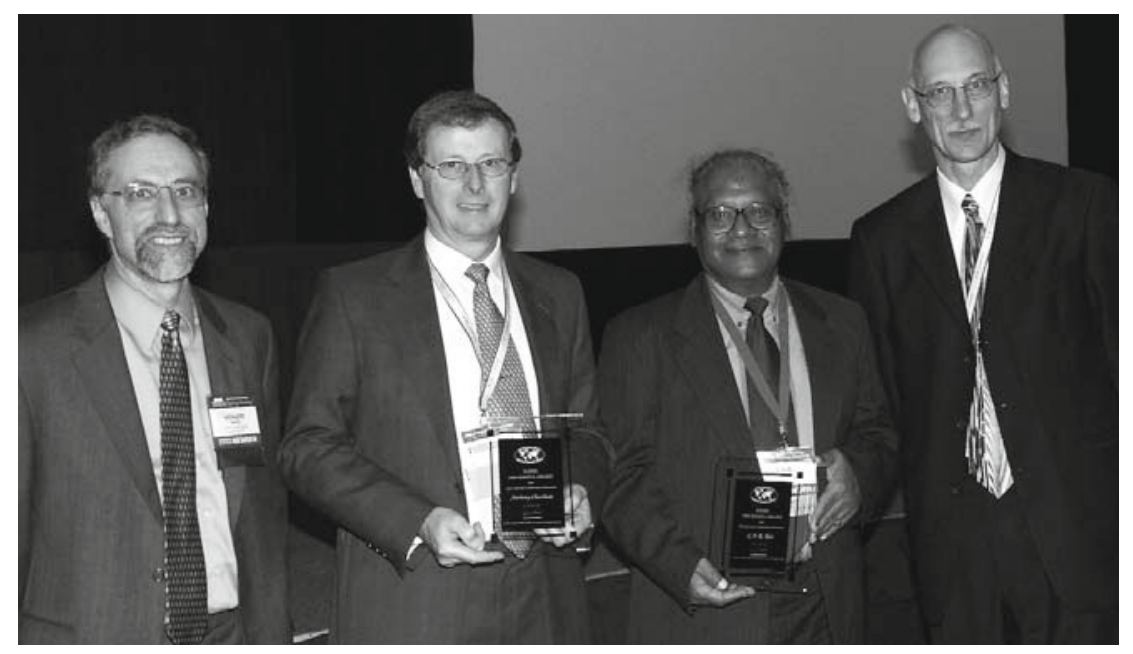

(Left to right): MRS President Howard Katz (Lucent Technologies), Sōmiya Award recipients Anthony Cheetham (UCSB) and C.N.R. Rao (JNCASR), IUMRS President Robert J. Nemanich (NCSU). 
tions versus complete dislocations. This discussion was initiated by the observation of dominance of partial dislocation emission in computer simulation in contrast to experimental results where complete dislocations appear even in materials with very small grain sizes. Swygenhoven hypothesized that the nucleation of partial dislocations is not governed by the stacking fault energy only, as recently proposed by Yamakov and co-workers. Instead, the ratio of unstable stacking fault energy over the stacking fault energy needs to be considered. Swygenhoven correlated the hypothesis to several computational results, including her own and that of other groups. Another important conclusion of her talk was that MD simulation cannot show the rate-limiting process of deformation, as the different mechanisms appearing in MD simulation provided that the applied stress is sufficiently large. This limitation of MD simulation is partly due to the time-scale limitation of the method. Swygenhoven concluded that simulation could only teach scientists what mechanism exists in principle, and therefore, experiments should be used to determine the rate-limiting processes and the conditions complementing computational effort.

The generation of a monodisperse assembly of nanoparticles typically requires an extensive size-selection process. However, as reported in Symposium M, T. Hyeon (Seoul Natl. Univ.) has developed methods to directly synthesize monodisperse iron nanoparticles. By taking advantage of the thermal decomposition of iron-surfactant complexes and by fully separating the nucleation process from the growth process, Hyeon and co-workers

\section{Poster Prizes Awarded at the 2004 MRS Spring Meeting}

The 2004 Materials Research Society Spring Meeting chairs awarded prizes for the best poster presentation. Prize recipients received $\$ 500$, a certificate, and the honor of having their posters displayed for the remainder of the Meeting. Posters and authors awarded prizes were (A9.21) Spectroscopic Ellipsometry Study of High Rate a-Si:H Growth by Means of the Remote Expanding Thermal Plasma and an Additional Ion Bombardment, A. Smets, E. Kessels, and R. van de Sanden (Eindhoven Univ. of Technology, The Netherlands); (F6.4) Effect of Aqueous Solution Chemistry on Accelerated Cracking of Nanoporous Thin-Films, E.P. Guyer and R.H. Dauskardt (Stanford Univ.); (I10.10) Synthesis and Transistor Performance of Tetracene Derivatives, H. Moon (Univ. of Michigan, Ann Arbor and Bell Labs, Lucent Technologies) and E.-J. Borkent, A.J. Lovinger, and Z. Bao (Bell Labs, Lucent Technologies); (M5.43) Synthesis of Hollow Cobalt Chalcogenides Nanostructures, Y. Yin and P. Alivisatos (Lawrence Berkeley National Laboratory); (M8.11) LocalField Effects on the Spontaneous Emission Rate of CdTe Quantum Dots in Dielectric Media, S. Wuister, C. de Mello Donega, and A. Meijerink (Utrecht University, The Netherlands); (W4.10) Glycera Jaws: A Biocomposite of Metals, Melanin and Proteins, D. Novak, H. Lichtenegger, J. Harreld, N. Slack, G. Stucky, and H. Waite (UCSB); and (Y5.18) Lithographically Patterned Microcoils for High Sensitivity NMR Chemical and Biological Detection, J.L. Herberg, S.C. Chinn, R. Maxwell, A.F. Bernhardt, and V. Malba (LLNL).

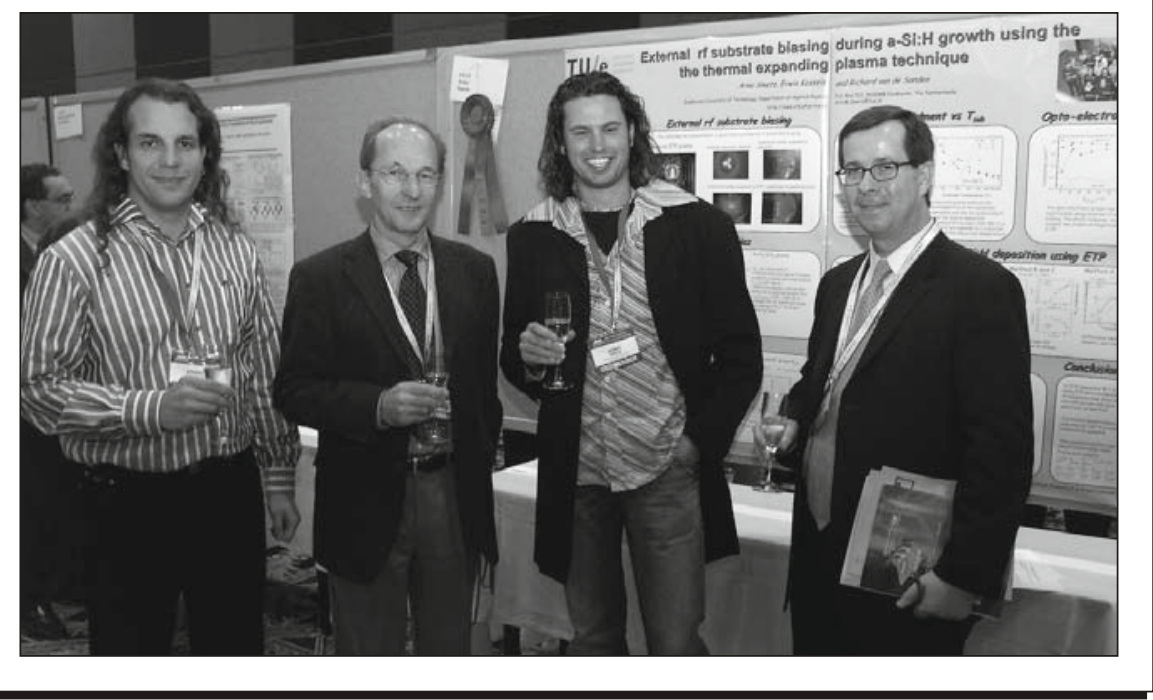

synthesized spherical, monodisperse, iron nanoparticles with controlled diameters in $1 \mathrm{~nm}$ increments from $4 \mathrm{~nm}$ to $16 \mathrm{~nm}$. Adding more surfactant and aging led to larger particles. Subsequent oxidation converted them to maghemite nanoparticles. Hyeon also reported on diametercontrolled synthesis of ferromagnetic and antiferromagnetic iron nanorods and nanowires and proposed growth mechanisms for these structures.

Also in Symposium M, Z.J. Zhang (Georgia Tech) examined the control of magnetic spinel ferrite nanoparticles and showed their biomedical applications. His focus was on how surface chemistry and magnetic anisotropy and superparamagnetic properties can be used to manipulate the size and shape of nanoparticles. He showed how cubic and spherical particles can be made due to differences in coercivity. His movie clip demonstrated how magnetic particles can be inserted into cells and the cells made to move through use of external magnets. Researchers are working toward biomedical applications such as intravenous injection and magnetically directing of particles to different places in the body.

N. Halas (Rice), in her Symposium R talk, "Rational Design of Plasmonic Nanoarchitectures," showed the tunability of plasmonic resonances in nanoshells and nanorods. Plasmonics is based on the configuration of electrons in nanoscale metals being affected by the shape of the metal structures, giving rise to strong optical resonances (plasmons). Halas addressed why spherical shells are so tunable, with a few nanometer changes in shell thickness leading to several hundred nanometer changes in wavelength. She described the plasmon configurations of the core and the shell and how their hybridization leads to strong splitting and these tunable effects. This model shows that as geometries are made more complex, the plasmon response can be interpreted as the mixing of plasmons of simpler geometries in analogy with the hydridization of electronic orbitals in molecular orbital theory.

A.K. Singh (SNL, Livermore) showed the possibilities for microfluidic devices by putting various materials in the microfluidic channels. In his talk in Symposium O, Singh showed that by filling the channels with monomers and masking part of the chip and photopolymerizing the monomer, patterns of porous materials or membranes can be made within the channels. This technology can improve on that currently used in order to make faster, better, and cheaper miniaturized separation and flow control 


\section{Graduate Students Receive Gold and Silver Awards}

During an awards ceremony held on April 14 at the 2004 Materials Research Society Spring Meeting, graduate student finalists received Gold and Silver Awards.

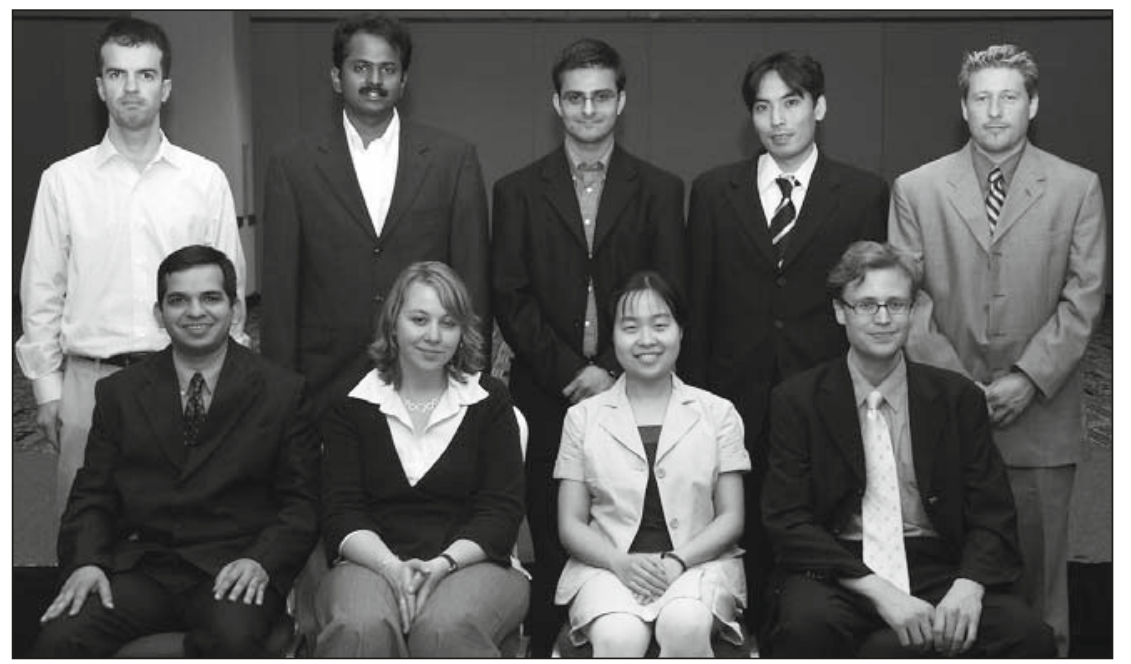

Gold Graduate Student Awards went to (first row, left to right): Rajaram A. Pai (University of Massachusetts, Amherst), Laura Vogelaar (University of Twente), Yuanijia Zhang (Cornell University), and Markus J. Buehler (Max-PlanckInstitute for Metals Research); (second row, left to right): Adamos Dalis (University of California, Los Angeles), Ragesh Puthenkovilakam (University of Calfornia, Los Angeles), Ali Javey (Stanford University), Kenji Nomura (Tokyo Institute of Technology), and Sascha Welz (Lawrence Berkeley National Laboratory). Not shown is Manish Deopura (Massachusetts Institute of Technology).

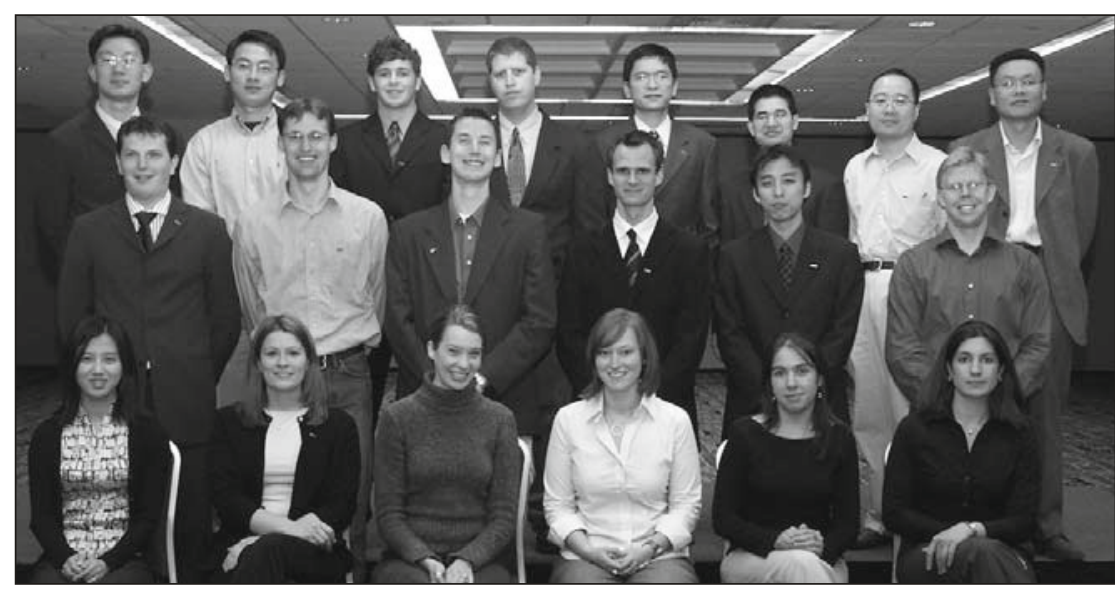

Silver Graduate Student Awards went to (first row, left to right): Wensha Yang (University of Wisconsin, Madison), Megan Pattison (Purdue University), Julie Casperson (California Institute of Technology), Hannah Storrie (Northwestern University), Maria Aboy (University of Valladolid), and Mitra Taheri (Carnegie Mellon University); (second row, left to right): Harold Jacobsen (Fraunhofer ISIT), Sander F. Wuister (Utrecht University), Byron McCaughey (Tulane University), Ulrich Rant (Technical University Munich), Yusuke Daiko (Nagoya Institute of Technology), and James M. Zahler (California Institute of Technology); (third row, left to right) Hyo Sik Chang (Kwangju Institute of Science and Technology), Yi Liu (University of California, Los Angeles), Philip Costanzo (University of California, Davis), Eli Rothenberg (Hebrew University of Jerusalem), Zhaohui Zhong (Harvard University), Nanfeng Zheng (University of California, Riverside), Ming Su (Northwestern University), and Hyoungsub Kim (Stanford University). Not shown are Benjamin B. Yellen (Drexel University) and Rachel Yotter (University of Washington). systems, for example, to create a chromatography device on a chip or to sort proteins by size.

Symposium A celebrated its 20th anniversary as an MRS Spring Meeting symposium dedicated to materials research on Si thin films. The precursor of the present day symposium, held in 1984, emphasized $\alpha-S i: H$ technology for thinfilm transistors; however, successive symposia have been broadened in scope. Topics now encompass many forms of thin-film and nanoscale $\mathrm{Si}$ as well as numerous applications. For example, in 2004, invited lectures were given on diverse subjects such as lasing action by Si:Er (A. Polman, FOM-Inst.); memory devices from Si nanoclusters (S.-J. Park, KJIST); and flexible electronics based on Si thin film (S. Wagner, Princeton); the latter jointly with Symposium I.

G. Cragnolino (Southwest Research Inst.), the first speaker in Symposium CC, gave a 25 -year overview on container materials for high-level radioactive waste. In a later session, R. Ewing (Univ. of Michigan), taking a phrase from the insurance industry, said, "You can't predict the future, but you can prepare," as he addressed the question of whether performance assessments of geological repositories for nuclear waste are necessary or sufficient. While the answer to that question differs depending on who is asked, it is clear that the issue of waste storage is complex, due to the complexity itself of the behavior of nuclear waste over thousands of years into the future and the political and regulatory environment in which decisions are made. Ewing said the study of models for nuclear waste can be compared to Vaucanson's mechanical duck of 1738. It looks like a duck, moves like a duck, eats like a duck, but is it a duck? Whether models are fully accurate representations of the likely outcome, there is much to be learned from them, and combining that with prudent guiding principles can help steer activity down the right path, for instance, seeking stable waste forms with slow release, employing multiple barriers, and allowing for margins of error. However, many pitfalls can be encountered along the way. Pointing out that although numbers come out of many models, Ewing suggested the results of models of nuclear waste behavior would be better used qualitatively to help direct strategies that make sense, rather than using the numbers simply to compare to regulatory limits.

For further details on the technical content of the Meeting, see the following symposium summaries. Proceedings are available on-line at www.mrs.org 


\section{0-Year Progress Seen in $\alpha-S i: H$ and Nanocrystalline Si Thin Film (See MRS Proceedings Volume 808)}

Symposium A, Amorphous and Nanocrystalline Silicon Science and Technology-2004, sponsored three special ses- sions. One session included reviews of the current scientific understanding of hydrogen in group IV semiconductors and its role in the metastability of $\alpha-\mathrm{Si}: \mathrm{H}$. Another session included reviews of current and future PV technologies. A third special session was retrospective in nature, celebrating the 20th anniversary of MRS Spring symposia dedicated to materials research on thin-film Si.

In the anniversary session, R.A. Street (PARC) reviewed the advances and the-

\section{Seminars Present Opportunities for U.S. Funding in Materials Research}

The U.S. government offers major funding thrusts in the areas of health-related research and hydrogen studies. To illustrate the increasing opportunities for materials research in these areas, several program directors from the U.S. NIH and a panel of presenters from the Department of Energy (DOE) presented seminars on these topics. Representatives from the NSF also presented a funding seminar, and the National Research Council (NRC) held a town meeting on smaller research facilities.

In January this year, officers of the Materials Research Society visited program directors at NIH in order to build a relationship on behalf of the MRS membership. One of the first outcomes of this visit was a seminar at the MRS Spring Meeting in which three program officers described the mission of NIH and how materials research fits in. In general, opportunities exist in NIH for biomaterials, including tissue engineering, drug-delivery, sensors, and nanoscience and nanotechnology. Opportunities reside in various institutes and consortiums within NIH.

Fei Wang, program director for the Biosensor and Platform Technology Scientific Program in the Division of Discovery Science and Technology in the NIBIB produced a grid identifying her institute (NIBIB) within the realm of NIH. While $\mathrm{NIH}$ institutes are predominantly concerned with diseases such as cancers in the National Cancer Institute, or on organs such as heart and lung in the National Heart, Lung, and Blood Institute, her institute focuses on biomedical imaging and bioengineering technologies, which is where materials research finds interactions with biomedical research.

Department of Energy (DOE) www.energy.gov www.hydrogen.gov

National Institutes of Health
BECON, the Bioengineering
Consortium
www.becon.nih.gov

National Institute of Biomedical Imaging and Bioengineering (NIBIB) www.nibib.nih.gov

National Institute of Dental and Craniofacial Research (NIDCR) www.nidr.nih.gov

National Research Council Committee on Smaller Facilities www7.nationalacademies.org/bpa/ projects_COSF_committee.html

Eleni Kousvelari, acting director of the Center for Biotechnology and Innovation at the National Institute of Dental and Craniofacial Research (NIDCR), said that the center seeks to develop the next generation of breakthrough biomedical technologies to improve oral health. To accelerate the technology development process, the center will rely on interdisciplinary research approaches. The challenges, she said, reside in the different languages spoken by clinicians, biologists, materials scientists, and engineers. The ongoing collaboration among the different scientific disciplines, she said, is essential.

Jeffery Schloss, program director for Technology Development Coordination at the National Human Genome Research Institute (NHGRI), discussed the NIH Bioengineering Consortium (BECON), which fosters support for bioengineering research. The consortium brings together many of the institutes, including his (NHGRI). Each of these institutes within the consortium receives its own appropriations from Congress. The consortium launches programs that involve the various institutes, encouraging a team approach. Opportunities reside here for materials scientists. Schloss emphasized-greatly-that materials researchers contact program directors for consultation before submitting proposals.

Numerous research funding opportunities exist within DOE in the areas of hydrogen production, storage, and fuel cells, as described by a panel of representatives: Harriet Kung (Basic Energy Sciences, DOE), Carole Read (Energy Efficiency and Renewable Energy, DOE), Susan Lesica (Nuclear Energy Science and Technology, DOE), Wayne Surdoval (DOE Office of Fossil Energy), and Kevin Hurst (White House Office of Science and Technology Policy). Mildred Dresselhaus (MIT) gave an overview of the hydrogen effort. She chaired a workshop last year that yielded an important report on the basic research requirements for moving forward in this area. The report also identified specific research areas and will be used as a roadmap for funding basic research solicitations in support of the President's Hydrogen Fuel Initiative. The panel members then spoke about specific basic and applied research opportunities in each of their programs.

In the NSF seminar, Lance Haworth of the Division of Materials Research (DMR) highlighted the wealth of funding opportunities in materials research. These opportunities are available throughout NSF, especially as the leading agency of the National Nanotechnology Initiative, a multiagency initiative. He said that support of materials ranges from fundamental phenomena to functional materials, systems, and devices. Both curiosity-driven and applications-driven research is funded. In FY03, NSF worked with a budget of $\$ 400$ million, \$250 million of which went to DMR. NSF is completing its 5-year plan on "Grand Challenges" in nanomaterials research, which focused on materials by design. It is now completing its recommendation for the next 5 years of "Grand Challenges," focusing on NanoFoundries. Haworth's most important advice is for scientists to contact program managers to help them determine how to direct their grant proposals.

The National Research Council Committee on Smaller Facilities, chaired by Robert Sinclair (Stanford Univ.), held a town hall meeting. Sinclair said that the challenge regarding smaller facilities is in determining what and where they are and how they help advance science. The questions his committee is asking include what are the best roles of smaller facilities and how can they be best supported, and should there be a national strategy for the establishment of facilities. He had questionnaires available at the seminar for facility managers and users. 
matic differences between the Spring symposia in 1984 and 2004. The focus of

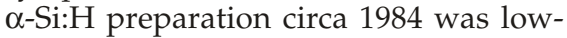
temperature rf PECVD, typically from pure $\mathrm{SiH}_{4}$. This material suffered from continuous light-induced degradation versus time by the Staebler-Wronski Effect (SWE), explained in terms of the generation of a single metastable defect, the $\mathrm{Si}$ dangling bond. In contrast, as emphasized by S. Guha (United Solar Ovonic), one "modern" form of rf PECVD $\alpha$-Si:H-optimized for stabilized PV performance-is prepared with enhanced $\mathrm{H}_{2}$-dilution of $\mathrm{SiH}_{4}$. Studies of " $\mathrm{H}_{2}$-diluted" materials reveal features-such as saturation of light-induced degradation versus timethat support a more complex SWE explanation with the involvement of at least two mechanisms and associated defect generation. The potential for further improvement of $\alpha-\mathrm{Si}: \mathrm{H}$ stability based on an in-depth understanding of the SWE will likely motivate future research on the nature of these mechanisms/defects.

In addition, Symposium A in 2004 emphasized $\alpha$-Si:H and nanocrystalline $\mathrm{Si}$ (ncSi:H) films equally. The counterintuitive observation that optimized ncSi:H is obtained with suppressed $\mathrm{H}_{2}$-dilution continues to withstand scrutiny. The desire to fabricate thick nc-Si:H has led to expanded research in alternative, highrate deposition methods; the challenges in scale-up were reviewed by J. Schmitt (Unaxis). Although a complete microscopic understanding of Si film growth mechanisms remains elusive, progress is being made (E.S. Aydil, UCSB), and in situ and real-time analysis provides guidance when operating within the complex deposition space of thin-film Si. One such report by A. Smets and colleagues (Eindhoven, The Netherlands) garnered a best poster award.

Symposium Support: PARC, United Solar Ovonic LLC, and Voltaix.

\section{Putting the Strain on Silicon Increases Carrier Mobility}

(See MRS Proceedings Volume 809)

Of all semiconductors currently used in electrical applications, Si shows the lowest electrical carrier mobility although it is the most widely used material in microelectronics. Symposium B focused on elementary and alloyed materials from group IV that show higher mobilities than plain Si: strained Si, strain relaxed buffer layers (SRBs), strained $\mathrm{SiGe}$, strained $\mathrm{Si}$ and $\mathrm{SiGe}$ on insulator (SSOI), Ge in bulk wafers and on insulator (GOI), and III/V compounds on Ge substrates. As such, the Symposium presented an overview of state-of-the-art techniques for fabricating these materials. Among these materials are superior thick Ge-graded SRBs and thin SRBs that use purposely injected point defects to enhance relaxation. Also, external stressinducing systems were covered, such as the use of a strained SiGe layer grown selectively in source and drain areas. Speakers discussed the merits of uniaxial versus biaxial strain and the theoretical background of why strain improves mobility, as well as some modeling work on channel strain induction by the selective growth of SiGe in source/drain areas. Various techniques for manufacturing of SSOI and GOI were presented, among which were the classical bonding and layer transfer techniques, but also some smart techniques were described, such as growth on free-standing structures (allowing elastic strain relaxation for defect-free SRB-like approaches) and liquid phase epitaxy of Ge in "microscopic crucible" structures on a Si wafer.

Defects are a main concern with these new materials, so a lot of attention went to characterization techniques such as defect etching, Raman and UV-VIS-IR spectroscopy, and electron beam-induced current. Two joint sessions were organized with Symposia C and D in order to highlight progress in two key technological areas: gate stack fabrication by deposition of dielectrics with high permittivity and shallow junction fabrication. This last topic also covered the important aspect of dopant and $\mathrm{Si} / \mathrm{Ge}$ interdiffusion. Some presenters also reviewed the state-of-theart of high-mobility devices such as MOSFET and MODFET transistors.

Symposium Support: AIXTRON AG, Applied Materials, ASM America, Hitachi, IBM Microelectronics, and UMICORE.

\section{New Processes Needed to Move Semiconductors to $45-\mathrm{nm}$ Node (See MRS Proceedings Volume 810)}

In Symposium C on Silicon Front-End Junction Formation-Physics and Technology, invited talks (A. Agarwal, Axcelis; J. Gelpey, Vortek; A. Jain, TI; and W.J. Taylor, Motorola) and contributed presentations highlighted the trends in research on the formation of ultrashallow junctions and their integration into devices. It was generally agreed that conventional RTP processes will be unable to reach the 45-nm node of the ITRS. As alternatives, concepts based on solidphase epitaxy or millisecond flash annealing and the use of impurities like fluorine or carbon were discussed.

\section{Peidong Yang Gives Outstanding Young Investigator Award Talk}

Peidong Yang (UC-Berkeley), who is the 2004 MRS Outstanding Young Investigator, gave his award talk as part of Symposium X. He gave an overview of his work on the chemistry and physics of semiconductor nanowires. Semiconductor nanowires are unique building blocks for nanoscale science and technology. Their anisotropic morphology can yield applications in a number of areas, including optoelectronics, photovoltaics, thermoelectrics, and sensing. A critical aspect to their applicability is rational growth and organization. Yang discussed various ways of aligning and assembling the nanowires. For example, he described the preparation of Langmuir-Blodgett films of nanowires, comparing it with floating logs on a river. Significant control over composition of growth has been achieved so far. He then discussed light emission from the onedimensional nanostructures as an application and the development of a nanowire and a quantum wire laser. Yang asserted that it is now becoming possible to develop various sub-wavelength optoelectronic

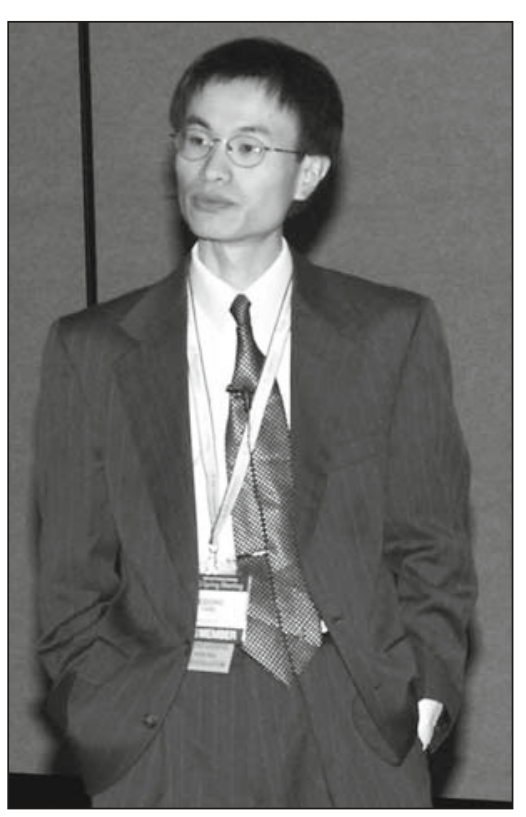
applications, including lasers, nonlinear optical mixing, photodetectors, and nanowire waveguides. He mentioned but did not detail energy-conversion applications including thermoelectrics and photovoltaics. 
An important consideration for future devices are silicides and germanides, which were discussed by J.A. Kittl (IMEC, Belgium) and several other speakers. Due to the trend to lower process temperatures, the scientific and technological interest was directed especially toward nickel silicides. Of similar importance for future technologies is the usage of silicon-germanium layers in which, as shown by N.E. Cowern (Univ. of Surrey), dopant redistribution is considerably affected by strain effects. Process development and optimization is hardly conceivable without technology computer-aided design. M.E. Law (Univ. of Florida) discussed the potential and limitations of process simulation. B. Colombeau (Univ. of Surrey, UK) and M. Hane (NEC) as well as several other presenters covered applications from atomistic modeling to the prediction of ultrashallow junction formation. The symposium was concluded by presentations of applications of state-of-theart characterization methods, commenced by W. Vandervorst (IMEC, Belgium) who demonstrated 2D carrier profiling on the nanometer scale.

Symposium Support: ASM International, Axcelis Tech., Philips Semiconductors Crolles, Varian Semiconductor Equipment Associates Europe BV, and Vortek Ind.

\section{Finer Points of High-K Insulators Examined}

(See MRS Proceedings Volume 811)

Symposium D on High- $\kappa$ Insulators and Ferroelectrics for Advanced Microelec- tronic Devices offered a tutorial in which the main electrical properties and issues of MOS devices with high- $\kappa$ gate dielectric stacks were reviewed. Several topics were covered, including charge transport mechanisms, generation of defects under electrical stress, time-dependent wear-out and dielectric breakdown, bias temperature instabilities, and poly-Si/high- $\kappa$ dielectric interaction issues. Future directions in the field were briefly mentioned, such as the integration of high- $\kappa$ dielectrics with highmobility substrates and metal gates.

During the technical program, one session was dedicated to the characterization of Hf-silicates and nitrided Hf-silicate layers, which are considered as the most promising candidates for the replacement of silicon oxynitride layers for the next generation of CMOS devices. The characterization and integration of ferroelectric layers, mostly for high-density memory applications (FRAM and FeFETs), were also presented. Other topics covered included the different deposition methods of high- $\kappa$ and ferroelectric materials; the physical, chemical, and electrical properties of these materials, also including reliability issues; and the integration of high- $\kappa$ gate dielectrics with metal gates. Also, a joint session was held with Symposium B on the deposition and characterization of high- $\kappa$ layers deposited on high-mobility substrates (SiGe and Ge).

Symposium Support: Applied Materials, Intel, Schumacher, TI, and Epichem Group.

\section{Universities Incorporate Nanotechnology into Undergraduate Curriculum}

Symposium BB on Educating Tomorrow's Materials Scientists and Engineers opened with a session in which speakers described efforts to incorporate nanotechnology in the classroom. As nanotechnology is seeing rapid advancements, acquiring funding from government agencies around the world, educators are creating ways to incorporate the topic in universities and precollege education.

M. Meyyappan, director of the Center for Nanotechnology at NASA Ames Center for Nanotechnology, described his center's affiliation with Santa Clara University. The NASA Center was established in FY1997, now employing about 60 people in various aspects of nanotechnology. Along with a research focus, the center has strong academic ties. Meyyappan said that while it is easy to incorporate nanotechnology in graduate studies and $\mathrm{PhD}$ programs, there is a problem in U.S. universities with incorporating this field at the undergraduate level. In order for undergraduate students to take an interest in this field, they want to see a large enough job market waiting for them.

Joe George Shapter (Flinders University of South Australia) described the success of teaching nanotechnology at the undergraduate level. In his program, a BS degree is given in nanotechnology in the honors program. This program offers students a strong solid science degree, enabling graduates to find jobs in the traditional areas and in new enterprises built from nanotechnology endeavors. Shapter defines nanotechnology as "the art of atomic or molecular control for the development of useful devices," emphasizing that the topic is not limited to a science, but expands to technology. The course, therefore, builds on various scientific disciplines (e.g., materials science, chemistry, and physics) and addresses topics of economics, commerce, law, and business.

\section{Oxide-Based Nanoelectronics Addresses Integration Challenges (See MRS Proceedings Volume 811)}

Presentations in Symposium E on Integration Challenges in Next-Generation Oxide-Based Nanoelectronics focused on materials science and engineering concerns related to oxide device fabrication such as deposition, patterning, interconnection, metallization, and large-scale integration to achieve high levels of device integration and multifunctionality on a chip. Some of the issues discussed were the difficulties and means to controllably grow epitaxial oxide films on technologically important substrates such as $\mathrm{Si}$ and $\mathrm{GaAs}$ and integration into existing technologies. Challenges described included the characterization of complex oxide structures and interfaces, and the growth of oxide-based nanostructures. The symposium showcased the range of potential applications for oxides from oxide electronics, use of oxides in future silicon technologies, to magnetic properties of oxide films. Among the presentations, S.J. Pearton (Univ. of Florida) covered a variety of oxide materials in his talk and presented critical issues concerning dry etching of these materials in order to use them in actual devices. V. Narayanan (IBM) discussed the challenges of replacing the silicon dioxide dielectric with high- $\kappa$ metal oxide insulators and metal gates for future CMOS technologies. J. Narayan (NCSU) described work on domain matching epitaxy (DME) where integral multiples of lattice planes are matched across the film-substrate interface for different materials systems.

Symposium Support: NCAET State Univ./NSF-Center for Advanced Materials and Smart Structures.

\section{Integrity of Porous Dielectrics and Longevity of Copper Interconnects Questioned \\ (See MRS Proceedings Volume 812)}

Symposium F, Materials, Technology, and Reliability for Advanced Interconnects and Low- $\kappa$ Dielectrics, presented advances and challenges for the development of interconnects for sub-90 nm BEOL technology. The keynote address by K. Maex (IMEC, Belgium) highlighted the "roadblocks" for integrating porous low- $\kappa$ materials and showed that there is currently no integratable material solution for $\kappa=2.4$ with a porosity value $<20 \%$. It was shown that the scalability of ultralow- $\kappa$ materials is limited due to an inability to seal mesoporous materials with porosity $>20 \%$ and because of the extensive sidewall damage, which occurs during photoresist ashing processes. The need for alternative integration schemes 
was suggested, and a process for forming airgaps in $\mathrm{SiO}_{2}$ was shown. The use of supercritical $\mathrm{CO}_{2}\left(\mathrm{scCO}_{2}\right)$ processes gained more attention this year than previously, and several speakers demonstrated advantages of the technology. A.J. Muscat (Univ. of Arizona) showed how, with $1 \%$ HMDS in $\mathrm{scCO}_{2}$, they can repair damaged low- $\kappa$ materials by reintroducing lost methyl groups, and R.A. Pai (Univ. of Massachusetts) showed how $\mathrm{scCO}_{2}$ can be used to extract porogens to obtain ordered mesoporous low- $\kappa$ films.

Sessions on copper reliability and metallization concluded the symposium. E. Zschech (AMD Saxony) demonstrated improvements in electromigration along the $\mathrm{Cu}$-dielectric barrier interface by locally alloying the $\mathrm{Cu}$ surface with $\mathrm{Al}$ prior to dielectric barrier deposition. M. Engelhardt (Infineon) discussed results that showed the resistivity requirements for copper wires will not be met for nanoscaled interconnects and stated that designs must be improved to continue improved performance with scaling. Two general materials questions remain: "Can an ultralow- $\kappa$ solution be found?" and "Is it possible that copper will be replaced for nanoscaled interconnects?"

Symposium Support: AMD Saxony, Dow Chemical Co., Intel, JSR Micro, LSI Logic, and Honeywell Electronic Materials.

\section{Efficient Injection of Spin-Polarized Electrons Is Focus of Spintronics}

In semiconductor spintronic devices, the spin of an electron is used as a unit of information in contrast to its charge in conventional electronics. In Symposium G, Semiconductor Spintronics, the building blocks required for a successful realization of devices and spintronic circuits were discussed.

A key issue for the realization of spintronic devices is the efficient injection of spin-polarized electrons into the semiconductor, for example, by using ferromagnetic semiconductor layers or ferromagnetic metallic layers. B.L. Gallagher (Univ. of Nottingham, UK) reported on the MBE of GaMnAs layers, with high conductivities and Curie temperatures up to $173 \mathrm{~K}$. The obtained Curie temperatures agree with the data obtained from $a b$ initio band structure calculations, as described by K. Sato (Research Center Jülich, Germany). In addition to the homogeneous doping with magnetic impurities, fabrication of digital ferromagnetic heterostructures was reported by E. Johnston-Halperin (Caltech) wherein electrical doping independent of the concentration of the magnetic dopants was achieved. Ferromagnetic properties of AlGaAs/GaAs 2D hole gases $\delta$-doped

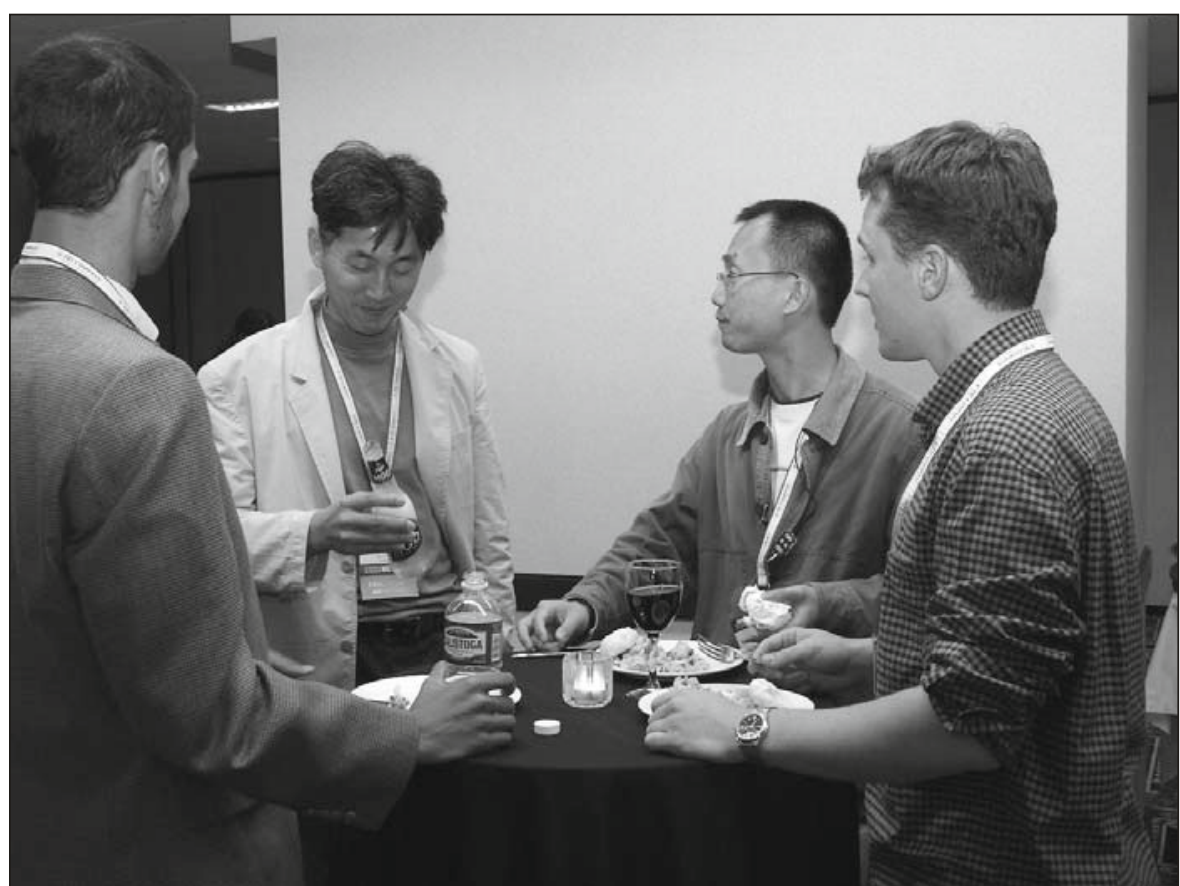

Close to 100 people attended the Student Mixer, providing student attendees with the opportunity to meet one another as well as other MRS members.

with Mn were also reported by M. Tanaka (Univ. of Tokyo, Japan). Here, the ferromagnetism could even be controlled by a gate electrode.

Spin manipulation in a semiconductor structure can be achieved by means of Rashba spin-orbit coupling. U. Zülicke (Massey Univ., New Zealand) discussed the Rashba effect in 2D hole gases, which is of particular importance in structures with $p$-type dilute magnetic semiconductors, such as GaMnAs, used as spin injectors. Based on the dependence of the spin-lifetime on the carrier concentration, X. Cartoixa (Univ. of Illinois) proposed a novel spin transistor structure that operates in the diffusive transport regime.

Symposium Support: Research Ctr. Jülich/Inst. of Thin Films and Interfaces, ONR/DARPA, and Rockwell Scientific.

\section{Hydrogen Has Profound Effects in Variety of Semiconductors (See MRS Proceedings Volume 813)}

Symposium H, Hydrogen in Semiconductors, brought together scientists studying diverse phenomena related to the presence of hydrogen in a wide range of host semiconductor materials. Zinc oxide, the first semiconductor in which hydrogen was studied, has been the subject of resurgent interest due to its potential optoelectronic applications.

Theoretical and experimental work has shown that isolated hydrogen acts as a shallow donor in $\mathrm{ZnO}$, although its defect structure is a matter of speculation. Since hydrogen could be an unintentional $n$-type contaminant and could passivate acceptors, its effects must be understood if $p$-type doping is to be realized. In GaAsN alloys, hydrogen effectively masks the effect of the nitrogen atoms, causing the material properties to revert to those of GaAs. As in the case of $\mathrm{ZnO}$, however, a vigorous debate ensued about the structure of the hydrogen-related complexes that are formed in GaAsN. Hydrogen in crystalline and amorphous silicon was discussed in a joint session with Symposium A. The effect and structure of hydrogen-related defects in silicon, including hydrogen molecules, were also discussed. There is also renewed interest in hydrogen platelets in exfoliation of semiconductor wafers in germanium. Similar experimental studies have extended these results to binary semiconductors such as InP.

Simulations and experiments on hydrogen in carbon nanotubes suggested an $\mathrm{H}$ storage capacity of $2-3 \mathrm{wt} \%$. Also, $n$-type conductivity by hydrogenation in boron-doped diamond was discussed. The $n$-type diamond is an excellent candidate for a low-barrier emitter, because the conduction band of the diamond is expected to be above the vacuum level. 


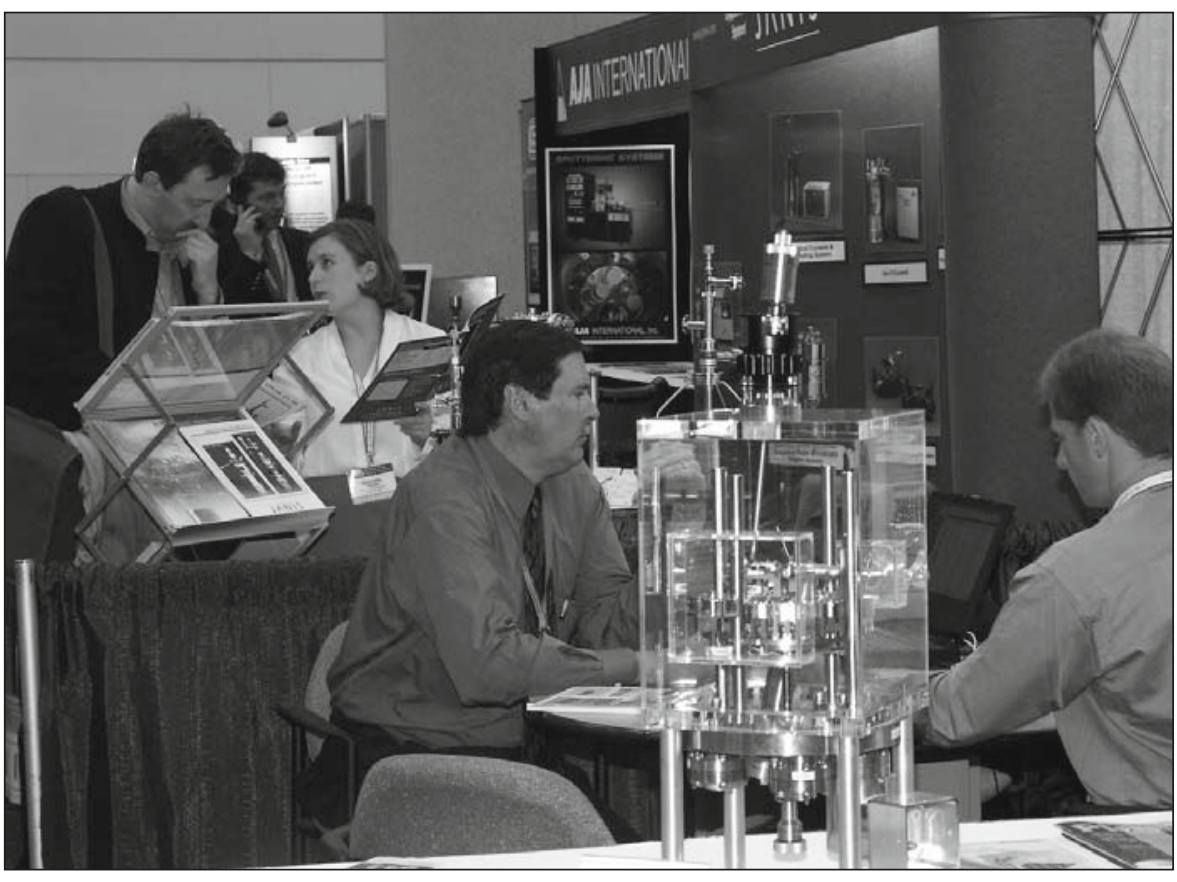

Exhibitors were on-hand to discuss their research equipment with Meeting attendees.

\section{Flexible Displays Move Toward Market Gains}

(See MRS Proceedings Volume 814)

The first market analysis on flexible displays was introduced by K. Allen (iSuppli/Stanford Resources). The world market is predicted to be $\$ 0.3$ million (in USD) in 2005, $\$ 1.2$ million in 2007, and to grow to $\$ 17.9$ million in 2010 . This is due to mobile applications such as mobile phones, PDAs, and smart cards because flexible displays are unbreakable and lightweight.

One of the advantages of flexible electronics is the ability to build a bendable device on a substrate. However, so far, there was no report on the OTFT performance measured as a function of bending radius. T. Sekitani (Tokyo Univ., Japan) measured the performance of a pentacene TFT as a function of bending radius, from which the strain can be obtained. Sekitani's group carried out many measurements on OTFTs with different polyimide (substrate) thicknesses and bending direction and achieved a universal law on bending durability. The OTFT is stable until its substrate is bent to $1.6 \%$ of strain.

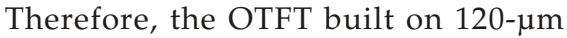
plastic can be bent down to the radius of $4 \mathrm{~mm}$ without inducing the track on the OTFT, and it decreases to $1 \mathrm{~mm}$ when the plastic thickness is $30 \mu \mathrm{m}$.

J.S. Kim and colleagues (Cambridge) proposed a trilayer structure for a polymer LED to increase the quantum effi- ciency and lifetime of the device. They inserted a thin conjugated polymer layer, a thin-film barrier, of 5-10 nm between poly (3,4-ethylenedioxythiophene): poly(styrene sulfonic acid) and LEP. Therefore, the PLED is a trilayer of cathode/light-emitting polymer/TFB/ PEDT:PSS/anode. The role of this layer is to block the electron injection to the PEDT:PSS layer and thus reduce the exciton quenching. The luminescence efficiency is greatly increased; for example, there is a threefold increase in red and green LEP. The researchers achieved a lifetime of longer than $50,000 \mathrm{~h}$ in green and red at $100 \mathrm{~cd} / \mathrm{m}^{2}$.

J. Lewis and colleagues (MCNC Research and Development Inst.) have developed an In/Ag/In flexible transparent conductor for displays. The insertion of an 8-12 nm silver layer between two ITO layers significantly reduces the sheet resistance along with a little decrease in transmittance. The film shows improved bending properties both as a function of radius and as a function of cycling. This can be a new TCO material for flexible displays.

A. Facchetti and colleagues (Northwestern) reported a high $n$-channel mobility OTFT using a material called DFHCO-4T, one of the carbon-based oligothiophenes. They achieved a fieldeffect mobility of $0.6 \mathrm{~cm}^{2} / \mathrm{Vs}$, on/off drain current ratio of $10^{7}$, and threshold voltage of $10 \mathrm{~V}$. This TFT is stable in air up to 30 minutes. The best $p$-channel mobility of organic TFT has reached to $5 \mathrm{~cm}^{2} / \mathrm{Vs}$ so far; however, the $n$-channel mobility is much lower. Therefore, many groups tried to modify the material to achieve high $n$-channel mobility in order to realize CMOS circuits based on OTFTs.

Symposium Support: MCNC Research and Development Institute and DARPA.

\section{Silicon Carbide Finds Role in Sensors and High Power Applications (See MRS Proceedings Volume 815)}

Silicon carbide $(\mathrm{SiC})$ is a very robust semiconductor material being actively developed for high power and temperature applications, especially in the field of power electronics and sensors for harsh environments. Symposium J was the third in a continuing series of symposia that covered $\mathrm{SiC}$ growth, defects, and devices. One of the sessions devoted to extended defects defined the current status of research regarding the degradation of SiC PiN diodes. P. Pirouz (CWRU) and R.E. Stahlbush (NRL) presented results obtained with electroluminescence and TEM, which significantly expand the understanding of this degradation process. M. Skowronski (CMU) presented results questioning the role of strain in this process. In one of the growth sessions, A.R. Powell (Cree) provided an update of the current state-of-the-art in substrate quality and showed evidence of considerable improvements in both micropipe and basal plane dislocation densities. H. Tsuchida (Central Research Institute of Electric Power Institute, Japan) discussed the step dynamics of micropipe closing and conditions for improved epilayers on both the carbon and silicon faces of $4 \mathrm{H}-\mathrm{SiC}$.

A.K. Agarwal (Cree) and P. Friedrichs (SiCED, Germany) presented industrial updates of $\mathrm{SiC}$ power device development where unipolar $4 \mathrm{H}-\mathrm{SiC}$ Schottky rectifiers continue to gain increasing market acceptance in power supply applications. Both speakers said that hybrid power switch modules (comprised of $\mathrm{Si}$ and $\mathrm{SiC}$ die combinations) are expected to play a significant role until improvements in $\mathrm{SiC}$ material quality and MOS process technology enable practical voltage-controlled switches based entirely on SiC. Improved understanding of processing and performance of $4 \mathrm{H}-\mathrm{SiC}$ MOS devices was reported by several speakers, including K. Fukuda (NIAIST), T. Kimoto (Kyoto Univ., Japan), and S. Krishnaswami (Cree).

The highlights of the session focusing on sensors involved the invited talks given by A.L. Spetz (Linkoping Univ.) and G.W. Hunter (NASA Glenn Research Center). State-of-the-art gas-sensing sys- 
tems based on $\mathrm{SiC}$ were presented, which are used for environmental monitoring in industrial settings, automobile exhausts, and even in the space shuttle. SiC sensors are preferred due to their high temperature stability and compatibility with microelectronics technologies.

Symposium Support: SULA Technologies and Cree Research.

\section{Understanding of Chemical-Mechanical Polishing Grows \\ (See MRS Proceedings Volume 816)}

Despite the fact that CMP has been in use centuries for glass polishing and more than a decade as a key technology in microelectronic manufacturing, a better understanding of the basic chemical and tribological processes is possible. The large number of variables that have to be considered for CMP were reflected in Symposium K on Advances in ChemicalMechanical Polishing.

Topics covered ranged from materials to be polished (e.g., $\mathrm{Cu}, \mathrm{W}, \mathrm{SiO}_{2}, \mathrm{Ni}, \mathrm{Ru}$, poly-Si, and polymers) to consumables such as pads (e.g., differently grooved, ceramic-coated, and fixed abrasive) and slurries (e.g., $\mathrm{SiO}_{2}, \mathrm{Al}_{2} \mathrm{O}_{3}, \mathrm{CeO}_{2}$, coated or doped, and polymer or $\mathrm{BN}$ as abrasive) to novel polishing methods and equipment such as using controlled high-pressure atmosphere, water-jet conditioning, or novel polishing head designs. Insight into the interaction between pad and substrate was presented by C.B. Rogers (Tufts). By using dual-emission laser fluorescence the extension of the slurry film can be photographed in situ on a microscopic scale.

Besides the applications in IC manufacturing, CMP is extensively used in MEMS fabrication and 3D integration and solves challenges in SOI-Si/Ge wafer preparation. A presentation by D.J. Stein (SNL) on a MEMS-based retinal prosthesis electrode array displayed the wide range of applications for CMP.

Several contributions were dedicated to modeling CMP. A review on modeling and design of consumables was given by D. Dornfeld (UC-Berkeley). G.P. Muldowney (Rodel, Inc.) presented detailed work to model the fluid transport and kinetics of CMP at the pad surface and in the pad groove. Others addressed the evolution of nanotopography, temperature effects in oxide CMP, STI, and copper modeling.

\section{Polymers Brighten Future for Nanophotonics}

(See MRS Proceedings Volume 817)

In a tutorial on nanophotonic materials and devices, for Symposium L, A. Polman (FOM-Institute, The Netherlands) and

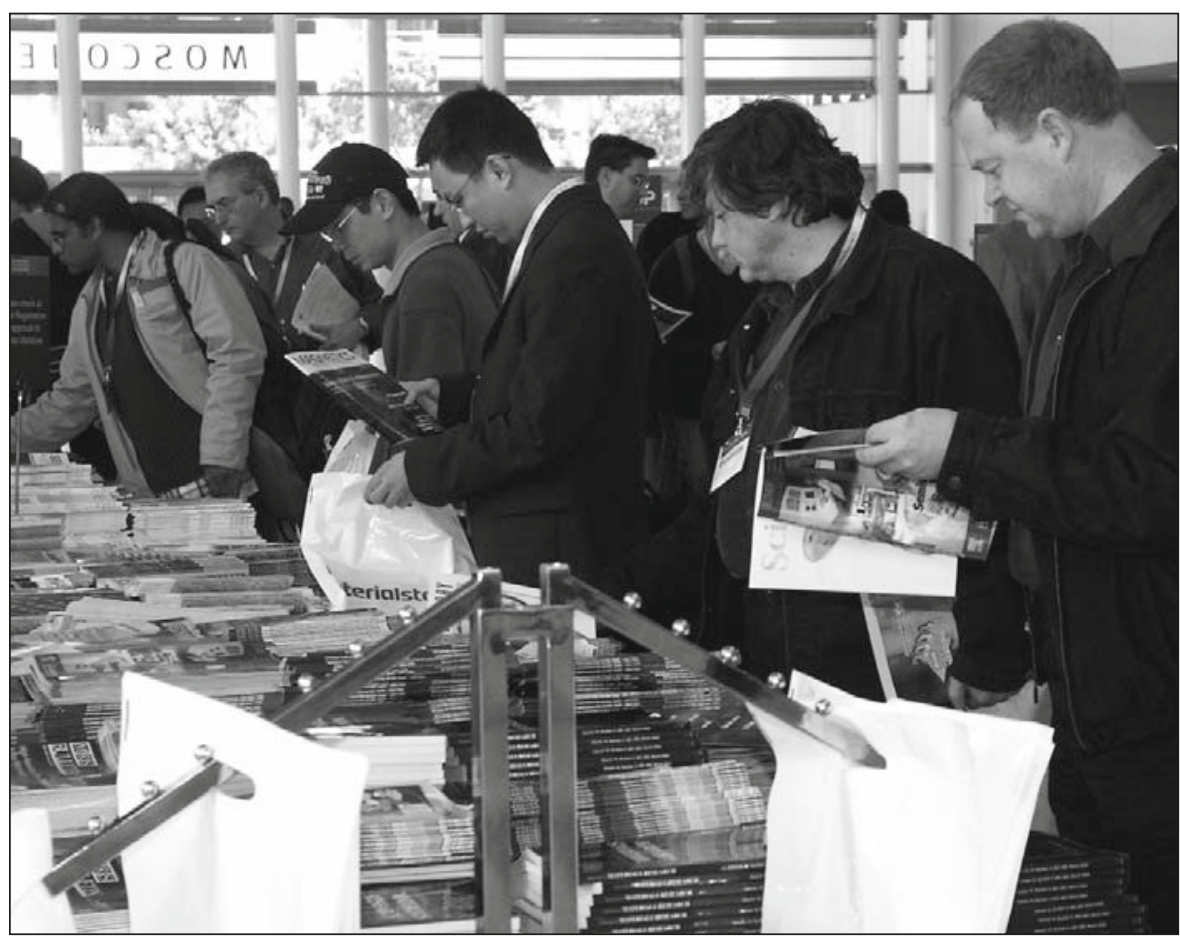

Attendees browse information at the MRS corporate affiliates' literature table.

S. Maier (Caltech) discussed dielectric and metallic photonic circuits in which light can be generated, guided, switched, and amplified.

During the technical sessions, E.A. Meulenkamp (Philips) and L.R. Dalton (Univ. of Washington) communicated exciting advances. Meulenkamp presented data on polymer-based light-emitting diodes, which cover the entire visible spectrum. These LEDs are deposited by an ink-jet type printing process. It is expected that the polymer LEDs will soon be commercialized for large-scale full-color displays. Dalton focused on the nonlinear and electrooptical properties of polymer-based integrated optical devices. He discussed the stability of these materials, especially for the near-infrared communication wavelengths, and he demonstrated very high figures of merit. The polymer integrated optics is close to the market as well.

P.B. Catrysse (Stanford) demonstrated how color filters can be integrated within a CCD device using the interconnect metals as plasmonic filters. This technique allows color filters to scale with increasing pixel density of a CCD array while at the same time increasing the resolution of a CCD array, and may be an example of a practical application of the budding plasmonics field.

\section{Nanoparticles and Nanowires Form Building Blocks for Biomedical and Other Applications \\ (See MRS Proceedings Volume 818)}

Symposium $\mathrm{M}$ on Nanoparticles and Nanowire Building Blocks-Synthesis, Processing, Characterization, and Theory provided an overview of the current successes and challenges in fabricating, characterizing, and manipulating nanomaterials, including dots, wires, rings, and assemblies of nanoparticles. Several talks presented very recent, unpublished experimental results, generating dynamic discussions on possible different interpretations. For example, F.M. Ross (IBM) introduced new measurements on semiconductor nanowire growth on $\mathrm{Au}$ catalysts.

The symposium covered synthesis and characterization of different inorganic nanomaterials, from magnetic nanostructures to metallic and semiconducting particles, and their use and assembly for various applications. These ranged from biological and biomedical applications (Z.J. Zhang, Georgia Tech; D.J. Norris, Univ. of Minnesota; N. Halas, Rice; and A. Belcher, MIT) to thin films for electronic devices (e.g., self-assembled magnetic thin-film devices described by C.T. Black of IBM and nanomaterials with new thermoelectric properties presented by A. Majumdar of UCBerkeley). 
Although the majority of talks were experimental, the theory contributions clearly showed the important role played by theory and computation in interpreting a rising sea of measurements (as highlighted by A. Zunger, NREL and J. Bernholc, NCSU, in the field of quantum dots and nanotubes, respectively). In addition, theory and computation are providing new foundations to understand complex phenomena such as transport at the nanoscale level, as described by J. Neaton (now at LBNL) and R. Car (Princeton), and inorganic/organic interfaces, discussed by J. Grossman (LLNL).

\section{Synthetic Grafts and Biosensors Emerge from Integration of Nanobiology with Biomaterials (See MRS Proceedings Volume 820)}

Symposium O, Advanced Microsystems-Integration with Nanotechnology and Biomaterials, covered nanofabrication devices and processes as well as their convergence with biological systems. The field of tissue engineering has been revolutionized by the insertion of microfabrication and nanofabrication tools, as described by T.A. Desai (Boston Univ.). Recent studies have shown that cell behavior in 3D culture is markedly differ-

\section{Novel Nucleation Theory Presented with Possible Application} to Sickle Cell Hemoglobin

Symposium Q on Nucleation Phenomena-Mechanisms, Dynamics, and Structure brought together researchers working in fields ranging from atmospheric science, through pharmaceutical, colloid, materials and surface sciences, to biomineralization, biotechnology, and medicine, who, in their work, are confronted by problems related to the nucleation of new phases. Experimental work presenting insights on the nucleation mechanisms of various systems were interspersed with theory discussing basic features of the nucleation processes as well as theory directly linked to intriguing experimental results. Simulations of systems ranged from well studied models to the complexity of freezing water.

During the intermissions, attendees continued discussing a novel mechanism of nucleation of ordered solid phases from solution by a metastable dense liquid intermediate (see Figure 1). Based on their respective experimental results, B. Garetz (Polytechnic Univ.) and O. Galkin (Univ. of Houston) discussed its applicability to crystallization from solution and to the polymerization of sickle cell hemoglobin, which underlies the deadly sickle cell anemia. Theories by A. Kolomeisky (Rice) and J.D. Gunton (Lehigh) addressed fundamental issues of this mechanism.

Other sets of experiments were presented by R. Strey (Universitaet zu Koeln, Germany), who is preparing to directly determine the rate of nucleation of argon droplets, which can be immediately compared to theory, and by D.A. Weitz (Harvard), whose group has carried out one of the only two determinations of the shape and structure of the critical clusters in a system undergoing a phase transition.

Particularly spectacular was the presentation of I. Ohmine (Nagoya Univ., Japan), who accompanied his simulations of the rearrangement of the system of hydrogen bonds upon water freezing with carefully chosen music pieces.

Symposium Support: NASA/Microgravity Science and Applications Division, NSF, and NIST/Center for Theoretical and Computational Materials Science.

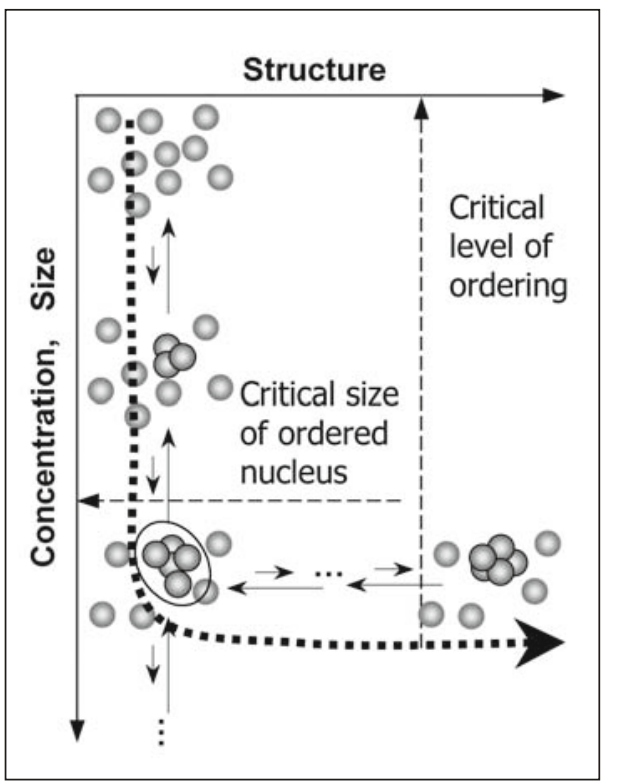

Figure 1. A schematic illustrating the formation of an ordered crystalline nucleus as a result of the superposition of density and structure fluctuations. (Courtesy of O. Galkin, University of Houston.) ent than that observed in conventional 2D petri dishes, and Desai demonstrated this phenomenon in cardiovascular tissues grown for the purposes of synthetic grafts. Micropatterned substrates were shown to promote physiological behavior in co-cultures of smooth muscle cells and endothelial cells in 3D polymer constructs. T.J. Webster (Purdue) utilized biodegradable scaffolds with nanotopographic features to promote the development of bladder tissues. The presence of nanodimensional features dramatically improved the properties of cultured bladder smooth muscle cells in terms of cell proliferation and collagen production.

Advances in nanoscale devices have led to greatly increased sensitivities for biosensors for DNA and biomolecular analysis. H. Craighead (Cornell) reported attogram-level resolution for nanomechanical resonators, detected by changes in the resonant frequency of the device as a result of analyte binding. A combination of nanofluidic structures with optical detection produces a novel means of confining the detection volume, thereby enabling extraordinarily sensitive detection limits for single molecule analysis. Methods involving a combination of silicon nanofabrication techniques with organic materials processing have led to further advances in biodetection.

Symposium Support: CIMIT, Draper Laboratory, Integrated Center for Nanotechnologies, $L A N L$, and SNL.

\section{Mechanical Behavior Built on Nanometer-Length Scales} (See MRS Proceedings Volume 821)

Symposium P on Nanoscale Materials and Modeling-Relations Among Processing, Microstructure, and Mechanical Properties highlighted the novel mechanical behavior at nanometer length scales using both experimental and theory/ modeling/simulation techniques. A broad range of material systems was covered, including thin films, metallic multilayers, nanodots and nanoporous materials, nanocomposites, and nanostructured metals and alloys. Dislocation glide-based strengthening mechanisms in passivated metal films on rigid substrates and nanoscale metallic multilayers were highlighted in several talks. W.D. Nix (Stanford) presented dislocation models of work hardening in passivated metal films and confined layer slip of nanoscale metallic multilayers. R.G. Hoagland (LANL) presented MD simulations that show that "weak" incoherent interfaces that are locally sheared by the stress field of a glide dislocation can be extremely resistant to slip transmission in the absence of a stress- 
concentration from a pile-up. F. Spaepen (Harvard) highlighted tensile data on thin metal films on polymer substrates and free-standing nanoscale metallic multilayers, while Z. Suo (Harvard) discussed fracture behavior of nanoscale metal films on polymer substrates. R.C. Cammarata (JHU) highlighted unusual internal friction and fatigue behavior of nanoscale metallic multilayers, while M. Dao (MIT) reported on the fatigue behavior of nanocrystalline metals.

Diffusional creep aspects of unpassivated metal film plasticity were discussed by H. Gao and T.J. Balk (MPI for Metals Research). Mechanical behavior of nanodots and nanoporous materials were described in presentations by W.W. Gerberich (Univ. of Minnesota) and C.A. Volkert (Forschungszentrum Karlsruhe, Germany), respectively. In the area of nanostructured metals, alloys, and nanocomposites, several presentations highlighted the state-of-theart experimental and modeling efforts. H. van Swygenhoven (Paul Scherrer Inst., Switzerland) emphasized bridging of in situ experiments such as deformation in a synchrotron source with MD simulations, J.R. Weertman (Northwestern) described stress relaxation experiments, and E. Ma (JHU) discussed the temperature and strain rate change experiments, coupled with electron microscopy, to elucidate the deformation mechanisms in nanocrystalline materials. The good combination of strength and toughness in nanocrystalline metals produced by severe plastic deformation (Y.T. Zhu and R.Z. Valiev, LANL) and in carbon nanotube-reinforced ceramic nanocomposites (A.K. Mukherjee, UCDavis) were also highlighted.

Symposium Support: NSF and Hysitron.

\section{Common Good Sought to Nanoengineer 3D Assemblies (See MRS Proceedings Volume 820)}

The topic of Symposium R on ThreeDimensional Nanoengineered Assemblies was not focused on a particular area of research, but on a goal that is being pursued by many research groups from a wide range of fields. Topics included areas that were "not quite truly $3 \mathrm{D}^{\text {" and }}$ "3D but not quite on the nanoscale level." Presentations addressed both the creation of these structures in materials and the phenomena that make possible unique applications for these materials.

Many approaches can be used to fabricate or assemble 3D structures with nanoscale precision, ranging from lithography, through beam-based direct write methods, through "soft lithography" con-

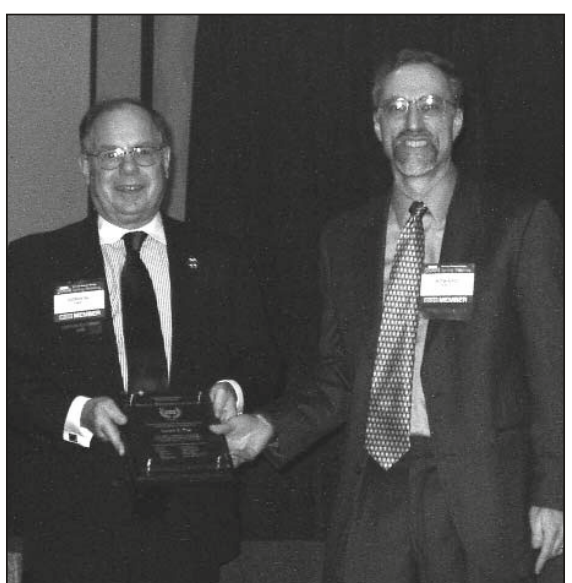

guides to control the flow of energy in a material, but this is too simplistic and limited a perspective. J.B. Pendry (Imperial College London, UK) has been one of the main proponents of the view that photonics and plasmonics are related on a fundamental level and that it is possible to model photonic crystals and other systems in terms of their plasmon resonances. Related phenomena include light funnels, magnetic properties from nonmagnetic materials, and left-handed index materials. Particularly inspiring talks in the area of photonics and plasmonics were given by E. Yablonovitch (UCLA), D.R. Smith (UCSD), H.A. Atwater (Caltech), and G. Shvets (Univ. of Texas-Austin).

Symposium Support: AFOSR and NSF.

Gordon Pike (left), who is the Editorin-Chief of the Journal of Materials Research (JMR), receives a special recognition award for sustained contributions over the years to MRS and for significant current contributions to JMR. MRS President Howard Katz (right) presented the award.

tact printing, through various pick-andplace methods to self-assembly. Selfassembly includes biological- and biomimetic-based methods, which generated significant interest when this Symposium was held two years ago, and this thread has continued through the current symposium. If biological processes can be understood and controlled or mimicked, then there is a potential to build structures starting on the nanoscale level. The directed self-assembly and the bottom-up approach to processing implicitly offers some 3D control from the nanoscale all the way to large structures. One session explored chemical conversion of biologically created calcium carbonate structures.

One of the most interesting aspects of nanotechnology is the ability to access physical phenomena that occur on the nanoscale. Many of these phenomena have become prototypical examples of the promise of new technology, from catalysis and fluidics to energy storage. Photonic systems are an important and well-known class of 3D nanoengineered systems. Two years ago, the emerging field of plasmonics received a lot of attention. This area has since become extremely important as the topic now spans several other symposia at the MRS Meeting. Nanoscale phenomena exploits the collective electronic excitations (plasma oscillations or plasmons) in solids or arrays of metal nanoparticles to manipulate energy and matter. Usually plasmonic systems are thought of in terms of specialty wave-

\section{Nanostructures Improve Alternative Energy Devices Due to Behavior at Surfaces and Interfaces (See MRS Proceedings Volume 822)}

Symposium $S$ provided an overview of how nanostructured materials can improve alternative energy devices. The main problem concerning new technologies of energy conversion and storage remains on the device's efficiency. Projects based on nanostructured materials can offer new or improved technologies in devices involving electrochemical reactions and heterogeneous catalysis such as fuel and solar cells, batteries, and so on. Nanoscale structures alter dramatically the surface reaction rates and electrical transport throughout the material, causing a dramatic improvement in energy storage, conversion, and generation. During the symposium, spectacular examples were shown to demonstrate the advantage of nanostructured materials to fabricate devices with superior performance. M. Graetzel (LPI Swiss Federal Institute of Technology, Switzlerland) discussed recent results about dye-sensitized solar cells (DSSC), showing devices with very good performance (efficiency higher than $11 \%)$. Clearly, modifications and new architectures of semiconductor nanostructured thin films (a fundamental part of DSSC and other solar cell devices) are the best way to improve solar cell performance. Significant results were also shown in the processing and performance of lithium-ion batteries and fuel cells. D.R. Rolison (NRL) discussed the processing and design of new nanostructured architectures in order to obtain lithium-ion batteries with higher performances than are currently available. The sol-gel process showed to be the best option in order to obtain such controlled nanostructures. Based on the presentations, the combina- 
tion between nanostructured materials and alternative energy devices is a promising way to develop a wide range of new technologies for a healthy future and is an active research area.

From CNTs to Organic Thin Films, Field of Molecular Electronics Moves Forward

Symposium T provided an overview of molecular electronics with numerous outstanding contributions in topics closely linked with the field. The scope ranged from carbon nanotubes, self-assembled molecular structures, nanometer-scale organic thin-film devices, and transistors to new concepts for the interface between solid-state and molecular structures.

Numerous methods and investigations of carbon nanotubes were presented, including their assembly on prestructured surfaces or into electronic circuits, their deposition and investigation on semiconducting substrates, and their tunable electronic and optoelectronic properties. Examples of supramolecular self-assembly as a powerful tool to organize nanoscale objects with promising electronic properties were presented by several speakers. For example, organic $\pi$-systems have been stacked by hydrogen bonds to form nanoscale pillars; they were assembled on surfaces to yield stripe-like structures or they were reacted with metal ions to form controlled hybrid rods. In many cases, these supramolecular structures displayed significant electronic and optical properties.

Organic thin-film transistors have been investigated in nanoscale gaps and their electronic properties have been investigated. New concepts and experiments to integrate single molecules in electronic circuits have been reported, providing important experimental understanding regarding the factors governing the electronic transport properties in molecular structures. The interface between molecules and metallic or semiconducting electrodes was investigated in several contributions and new concepts for future device architectures have been presented.

Numerous theoretical models to predict electronic properties of nanoscale objects

\section{Materials Enter Quantum Computing}

As limits in the development of classical computing appear to be subject to Moore's law, research interests are turning to developments in quantum technology Plenary speaker Luiz Davidovich (Universidade Federal do Rio de Janeiro, Brazil) described various motivational factors for the development of quantum computing, which include the factorization problem that could break current cryptographic schemes and data search techniques. The basis of quantum computing, he said, is the qubit, which is the quantum equivalent of the bit. It is a unit vector in 2D Hilbert space, Davidovich said. Potential functions to benefit from quantum computing, he said, include ion traps, quantum dots, Josephson junctions, nuclear magnetic resonance, silicon, and optical lattices wherein atoms are trapped in 3D standing waves. All of these systems share the major problem of decoherence.

Bruce Kane (Univ. of Maryland), in his Symposium X talk on Si-based quantum computing, addressed this problem of decoherence in the example of semiconductor qubits, which decohere rapidly. Kane said that many quantum logic operations must be performed on a qubit before decoherence occurs. He discussed details of possible Si quantum computing systems that have been suggested in recent literature. Si appears to be a nearly ideal material for spin quantum computing. Devices demonstrating single electron spin manipulation and measurement are difficult but appear to be doable. There are, however, serious doubts about the ability to scale simple quantum logical devices into a technologically relevant quantum computer. Kane stressed that in spite of this somewhat pessimistic outlook, significant opportunities exist for materials research and nanofabrication at the end point of Moore's law scaling.

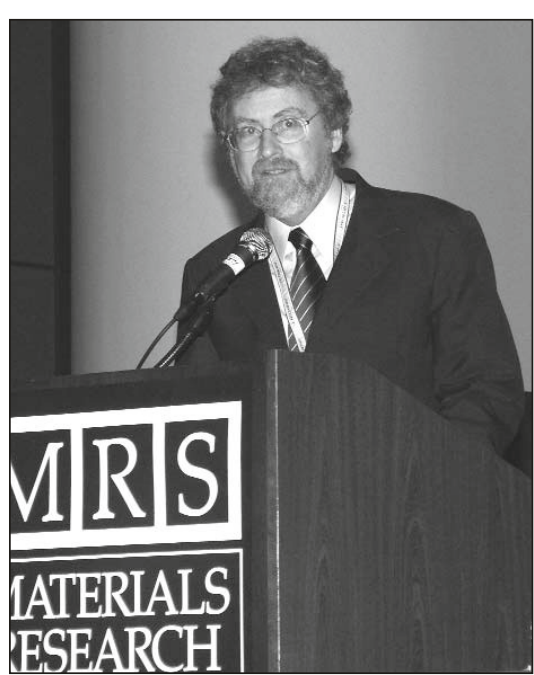

Plenary speaker Luiz Davidovich of the Universidade Federal do Rio de Janeiro, Brazil. have been presented and their predictive power has been discussed by comparison with the emerging amount of experimental results. In particular, both theoretical and experimental studies of inelastic and resonant electron tunneling spectroscopy are providing important evidence and insight into molecular junctions.

\section{Printing Isn't Just for Ink Anymore:}

OLEDs and Biological Materials Printed

Symposium $U$ focused on printing of functional materials, covering a broad cross section of work ranging from printing methods and materials to the characterization of printed devices and circuits. While a large number of presentations described the development of printed organic electronic devices such as transistors and OLEDs, several presentations outlined new developments in the direct writing and printing of biological materials.

A significant feature of the symposium was the breadth of printing methods that were discussed. In addition to new inkjet deposition and patterning results, new approaches such as nanoxerographic methods (H.O. Jacobs, Univ. of Minnesota; G.M. Whitesides, Harvard), dip-pen methods (C.A. Mirkin, Northwestern; P.V. Schwartz, CalPoly), and nanoimprinting were also discussed.

\section{Specificity of Proteins Opens Route to Design of Functional Materials}

Synthetic protein materials are envisioned as having a transformative impact in materials science applications as a consequence of the structural and functional specificity that are characteristic of these biosynthetic macromolecules. Several themes were developed in Symposium V on Proteins as Materials in order to explore the implications of this concept, in particular, with respect to the design of novel, functional materials based on polypeptide sequences for directed applications in medicine and nanotechnology. The first session delved into the design of structurally defined supramolecular assemblies based on oligopeptide folding motifs. Several lectures were presented that indicated that protein modules could be designed that were competent for selfassembly into nanoscopic objects (J.E. Padilla, UCLA), long-aspect ratio synthetic fibrils (D.N. Woolfson, Univ. of Sussex, UK; Y. Zimenkov, Emory), nanotubes (K. Lu, Emory), and hydrogel networks (J.P. Schneider, Univ. of Delaware). The following session dealt with integration of protein-based materials into devices. Practical applications of these devices were discussed with respect to fabrication of microarrays 
(J.A. Camarero, LLNL) and in DNA sequencing analyses (J.-I. Won, Northwestern). The final session focused on bioengineering of protein-based materials. The development of materials for tissueengineering applications based on native silk proteins (D.L. Kaplan, Tufts) and synthetic biopolymers (G. He, Univ. of Illinois-Chicago) were discussed in terms of basic protein design principles. The Symposium concluded with lectures on more advanced bioengineering techniques based on combinatorial protein libraries (M. Hecht, Princeton) and expansion of the genetic code (D. Tirrell, Caltech) that pointed toward new directions in protein-based materials design.

Symposium Support: NIH/NIBIB.

\section{Bio-Inspired Self-Assembly Leads to Muscle-Powered Microrobots (See MRS Proceeings Volume 823)}

Progress in bio-inspired materials research in the past decade is gradually changing the way materials and devices are made. Symposium W, Biological and Bio-Inspired Materials and Devices, outlined the latest development in this active field. Topics covered biological materials, biomineralization, self-assembly, bioinspired devices, biomimetic processing, and tissue engineering.

Self-assembly still leads the way of materials design and new concepts/ideas continue to emerge. In the joint session with Symposium O, G.M. Whitesides (Harvard) demonstrated the concept of designing functional, self-assembling systems. Z. Suo (Harvard) introduced the idea of a molecular car by moving assembled molecules with external field. Novel biomaterials and biodevices have been developed based on self-assembly, as were discussed by S. Zhang (MIT) and D. Pochan (Univ. of Delaware) (e.g., hydrogels with tunable rigidity). The integration of self-assembly with microprocessing technology opens a new direction in biodevices. An inspiring talk was given by graduate student J. Xi (UCLA) whose team self-assembled muscle cells onto MEMS and turned them into musclepowered microrobots.

There is also a trend that novel biomaterials and microfabrication are integrated together in the field of tissue engineering. J.T. Borenstein (Draper Laboratory) discussed the recent progress of using microfabrication to build 3D biodegradable materials suitable for engineering blood vessel networks. These networks are designed to replicate the critical fluid dynamic properties of physiological systems such as the microcirculation within a vital organ.

\section{Chemical and Biological Detectors Get Smarter and More Nosy}

Symposium Y on Materials, Mechanisms, and Systems for Chemical and Biological Detection and Remediation highlighted the major advancements in this rapidly growing field. Key detection systems ranged from MEMS and NEMS to photoacoustic to ion mobility mass spectrometers. T. Thundat (ORNL) reviewed the nanomechanics of microcantilever-based detection schemes. R. Berger (MPI, Germany) provided an overview of the exponentially growing field of cantileverbased sensors and devices. A. Boisen (Technical Univ. of Denmark, Lyngby, Denmark) discussed the cantilever sensors with integrated readout. A. Majumdar (UC-Berkeley) discussed real-time detection of biomolecules with a novel multiplexed readout method. Majumdar's technique can readout over 500 cantilevers simultaneously (see Figure 1). A MEMS-based correlation radiometer for chemical sensing was discussed by M.B. Sinclair (SNL). G.S. Shekhawat and V.P. Dravid of Northwestern University presented their innovative signal transduction approach based on embedded MOSFET in microcantilevers. This approach has the potential to meet the advantages of optical detection but without lasers, optics, and alignment, and it is readily amenable to the mass-production compatible MOS process.

Materials and processing challenges related to MEMS-based micro-gas chromatography were discussed by E.T. Zellers (Univ. of Michigan). N.S. Lewis (Caltech) discussed electronic nose from arrays of polymer composites. M. Sailor (UCSD) presented self-assembling, self-orienting "smart dust" sensors. M.J. Sepaniac (Univ. of Tennessee) presented noble metal-polymer nanocomposites as a versatile material for sensing and microfluidic separations.

Development of a miniature ion mobility spectrometer was discussed by K.B. Pfeifer (SNL). Carbon nanofiber interface to a molecular-scale process was discussed by M.L. Simpson (ORNL). P.M. Pellegrino (ARL) presented a novel photoacoustic chemical sensor using micromachined structures.

The symposium also included a poster session where many different aspects of detection mechanisms, techniques, and materials were presented. A poster presented by Julie Herberg (LLNL) on lithographically patterned microcoils for highsensitivity NMR for biological detection received a Poster Award. Silver Graduate Student Award recipient M. Su (Northwestern) presented a poster on directed assembly of nanomaterials for miniaturized chemical sensors.

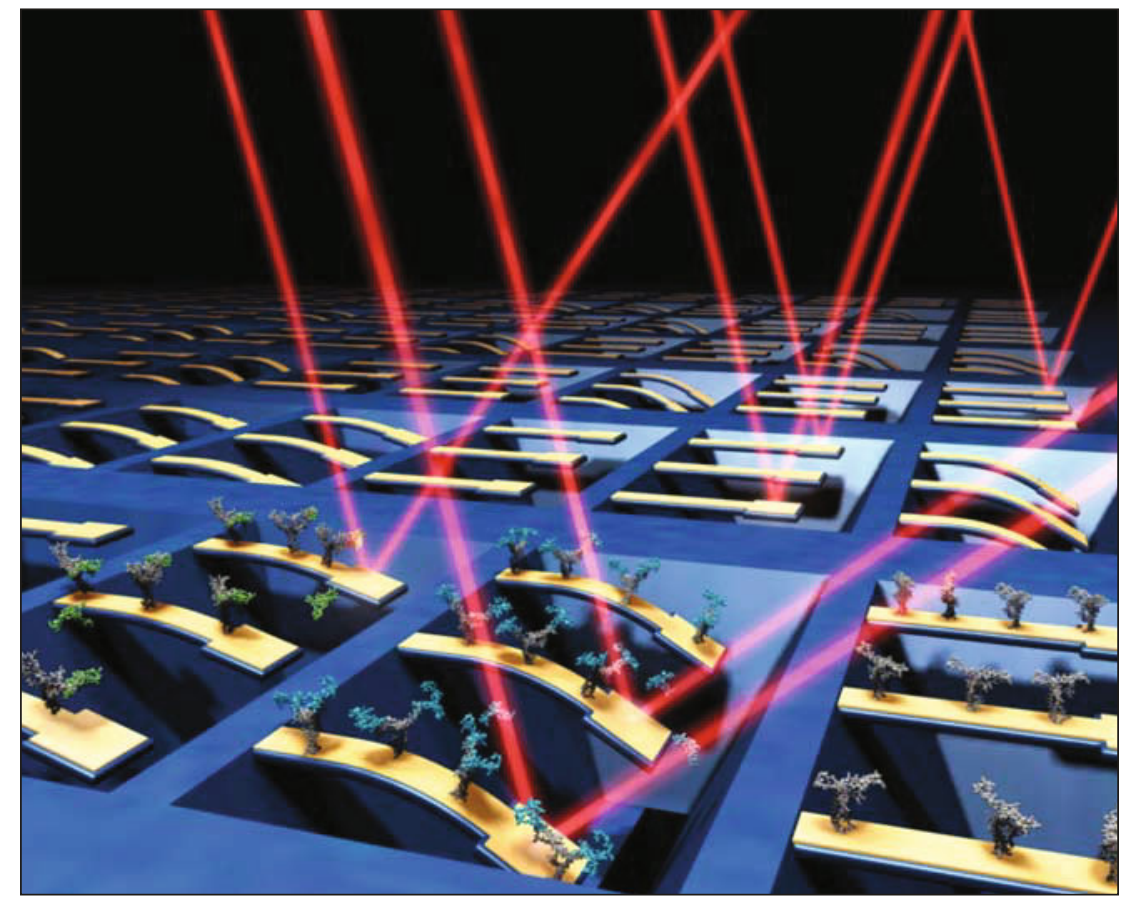

Figure 1. Schematic representation of a multicantilever detection scheme. (Courtesy of Arun Majumdar, University of California-Berkeley.) 
While more and more lessons from biology are being applied to real materials design and processing, scientists continue to search for new inspiration from the biological world. An interesting example was the hagfish discussed by J.M. Gosline (Univ. of British Columbia, Canada). The hagfishes are renowned for their ability to produce vast quantities of slime when provoked. The slime comes out of the glands in a concentrated form, but quickly swells and turns the surrounding seawater into a gel. When stretched, the fine slime fibers would undergo phase transformation into a silklike structure.

Symposium Support: NIH/NIBIB, LLNL, and The Whitaker Foundation.

\section{Symposium X Provides Overviews on CMOS Technology, Sports, and Biomaterials}

Presentations in Symposium X on Frontiers of Materials Research are designed to provide overviews of specific fields for the nonspecialist. At the 2004 MRS Spring Meeting, speakers presented talks on CMOS technology, sports, and biomaterials, among other topics.

Silicon-based CMOS technology has been the basis for microelectronic circuits and devices over the past three decades. Wilfried Vandervorst (IMEC, Belgium) described the increasing use of newer materials over the past 30 years, beginning with three materials $\left(\mathrm{Si}, \mathrm{SiO}_{2}\right.$, and $\left.\mathrm{Al}\right)$ to the present vast selection of materials in use, such as high- $\mathrm{\kappa}$ materials to replace $\mathrm{SiO}_{2}$. The limitations in scaling by the use of poly-Si as the gate material are being overcome by the use of metal gate electrode materials. Other tricks are also being used for enhanced performance such as the use of stressed Si layers in the channel region to obtain increased mobilities. The ultimate step would be the use of Ge instead of $\mathrm{Si}$ as the substrate.

Materials used in sports have unique functionality and performance requirements, and due to the competitive nature of sports, materials development in this area tends to be innovative as well as risk-taking as compared to the incremental steps of development that occurs in areas such as aerospace and automobiles, in which significant capital is involved. Jan-Anders Manson (EPFL, Switzerland), in his presentation on materials and sports, gave examples of how structural performances and functions are widely integrated in much sport equipment. For instance, the latest tennis rackets use these novel materials to reach the otherwise impossible combination of high stiffness, low weight, and extreme damping performance. Manson also described his role in materials investigations relating to the America's Cup.

In a presentation on bio-related materials, Samuel Stupp (Northwestern Univ.) said that the next frontier in medical technology is regenerative medicine. Materials play a role by inducing regeneration when it is not spontaneous; these materials themselves, however, do not replace tissues and organs. Stupp gave an example of research done on the regeneration of a spinal cord. A matrix of selfassembled nanofibers was loaded with neural cells that, within 24 hours, became neurons. The ultimate goal, he said, is to be able to inject a liquid into the spinal cord after an injury for complete repair.

Optical tweezers, by which light pins and controls the position of tiny beads, has enabled Steven M. Block (Stanford Univ.) to measure the motion of proteins tethered to beads to within nanoscale dimensions, approaching the measurement of a single base pair (3-4 $\AA$ ) in real time. One mystery Block put forth was whether the mechanoenzyme kinesin "walks hand-over-hand or like an inch worm?" Kinesin is a protein that behaves like a tiny motor. Its two heads terminating a coiled chain crawl along the length of microtubules, capable of carrying a bead along for the journey. The "motor protein" moves in discrete steps, but with an asymmetry in even and odd steps, as if walking with a limp. Block pegged this limp to the heads moving one over the other, but being more like two left feet going a different distance on each side. He attributed this asymmetry to biochemical states with different molecular conformations, which needs to be "undone" by one "foot" relative to the other. Block also talked about RNA polymerase (RNAP), which is a large protein complex at the heart of gene regulation and control.

Also in Symposium X, Peidong Yang (UC-Berkeley), recipient of the MRS Outstanding Young Investigator award, gave his award talk, and Bruce Kane (Univ. of Maryland) gave a talk regarding quantum computing. See the respective Sidebars for more on their presentations.

\section{Biological and Inorganic Materials Work Together}

The presentations in Symposium Z, Hybrid Biological-Inorganic Interfaces, covered a broad spectrum of research topics ranging from biomaterials to bioelectronics. In the area of biomaterials, S. Stupp (Northwestern) presented an overview of his group's efforts to use peptide amphiphiles that self-assemble into long collagen-like fibers as scaffolds to direct mineralization. This system, having been shown to direct mineralization in biological media, holds promise for bone regeneration. K. HamadSchifferli (MIT) discussed her work on the use of inductive heating as a "remote-control" means of tuning the functioning of biomolecules. In this approach, localized heating of a biomolecule is directed through an "antenna" comprising a tethered gold nanoparticle coupled to the molecule. Other research in the general area of external control of functional nanocomposites was presented by D. Hay (ANL), who reported that poly( $N$-isopropylacrylamide)-lipid-based materials mimic biological systems in that subtle changes in temperature trigger reversible changes in their structure, fluidity, and optical properties. These materials have also been found to serve as "molecular scaffolds," allowing for the incorporation and organization of inorganic nanoparticles such as quantum dots. The temperature-dependent structure of the scaffold can be used as a way to externally control (i.e., "tune") the packing arrangement and, therefore, the collective properties of the encapsulated quantum dots.

Several presentations on work done at the University of Wisconsin, Argonne National Laboratory, and Heriot-Watt University (Edinburgh, UK) described theoretical and experimental efforts directed at adapting synthetic diamond thin films for use as a platform for biosensors and bioelectronic devices. Surface-functionalized micro- and nanostructured diamond thin films possess properties well suited for biochemical/ biomedical applications, such as MEMScompatible chemical sensors, chip-based biosensors, implants, and bioelectrodes. Each of the three research groups has independently developed a photochemical (W. Yang, Univ. of Wisconsin), electrochemical (J. Wang, ANL), or oxidativepretreatment (P. John, Heriot-Watt Univ.) approach to the formation of monolayers through which oligonucleotides, hemeproteins, or FAD can be coupled to the diamond surface. Control of the surface chemistry of the diamond, in particular, 
its hydrophobicity, surface charge, and functionalization, is a crucial first step toward developing means for the selective coupling of biomolecules, while suppressing biofouling.

Symposium Support: LLNL/Chemistry $\mathcal{E}$ Materials Science Directorate and ANL/Center for Nanoscale Materials.

\section{Nanoparticles, QDs Probe into Life Sciences}

Symposium AA, Applications of Novel Luminescent Probes in Life Sciences, covered novel upconverting nanoparticles, long-lived lanthanide probes, semiconductor nanoparticles, quantum-dot-organic fluorophore hybrids, light scattering probes, genetically designed fluorescent proteins, and chemical derivatives of organic fluorophores. Partial solutions to lifetime discriminations, suppression of irreversible photobleaching, size, and biofunctionalization were presented, but it remains to be seen if all these properties will eventually be integrated into a single do-it-all probe. The general research community is likely to grow, now that probe development has been identified by the U.S. National Institutes of Health, as well as the Office of Science of the U.S. Department of Energy, as research areas of the future.

The Symposium was opened by M. Schaeferling (Univ. of Regensburg, Germany) who discussed the development of lifetime probes based on europium nanoparticles as immunoprobes, and the detection of oxygen, oxidases, catalases, and peroxidases. H.M. Mattoussi (NRL) presented his approach to nanoscale sensing by creating quantum dot-fluorophore hybrids, which work through fluorescence resonance energy transfer. W.E. Moerner (Stanford) presented his most current results on singlemolecule fluorescence detection, describing in detail a novel organic fluorophore that he derived from a photochromic dye. G. Bazan (UCSB) described his approach to DNA detection by creating peptidenucleic-acid-conjugated polymer hybrids that can amplify the fluorescence of regular organic dyes by orders of magnitude by exploiting the much larger fluorescence excitation cross section of the conjugated polymer. A.J. Fischer (UCDavis) then discussed applications and development of engineered phytochromes based on the expression of random mutations of biliproteins.

N. Halas (Rice) described the optical properties of nanoshells, which are nanoengineered light-scattering particles that show intense enhancement of Raman scattering and can deliver heat when tar- geted to cancer cells. C.E. Talley (LLNL) discussed the development of novel chemical nanosensors for $\mathrm{pH}$ and other metabolic indicators, based on surfaceenhanced Raman scattering. J. Zhang (UC-Santa Cruz) described the synthesis of metal nanoparticle aggregates as efficient enhancers of Raman scattering. $\mathrm{H}$. Vogel (EPFL) described the targeting and study of G-protein coupled receptors in synthetic membranes, vesicles, and live cells by selective labeling with fluorescent probes.
Symposium Support: LLNL/Chemistry and Materials Science Directorate, Quantum Dot Corp., and F. Hoffman-La Roche.

\section{Enhancements to Materials Education Explored}

Symposium BB on Educating Tomorrow's Materials Scientists and Engineers explored pressing issues in materials science education from elementary school education and outreach to advanced undergraduate courses and new opportunities for graduate teaching fellows.

\section{Chemistry of Spent Nuclear Fuel Gives Clues to Its Ultimate Storage (See MRS Proceedings Volume 824)}

In the opening talk in Symposium CC on Scientific Basis for Nuclear Waste Management, G. Cragnolino (Southwest Research Inst.) gave an overview of developments over the past 25 years in container materials for high-level radioactive waste. In the next session, L.O. Werme (SKB, Stockholm, Sweden) presented a comprehensive overview of the historical development on several aspects of studies of spent nuclear fuel.

J.A. Fortner (ANL) discussed results from a study of crystal chemistry of technetium and molybdenum in spent nuclear fuel and the role of these elements on release estimates of other radionuclides (see Figure 1$)$. Results from long-term ( $~ 8$ years) corrosion tests suggest that a fraction of the inventory of Tc in spent nuclear fuel may not dissolve when the fuel's $\mathrm{UO}_{2}$ matrix is oxidized and converted to U(VI) alteration phases. Fortner proposed that the Tc does not dissolve because a significant fraction of the Tc is incorporated into noble-metal-alloy epsilon particles, which can be found near the surface of corroded fuel fragments in the presence of uranyl alteration products (see Figure 1). R.J. Finch (ANL) spoke on the roles and fate of actinides during spent fuel alteration, in which he compared expectations of alteration products and actually observed secondary phases that will ultimately control the solubility of actinides in a repository.

The session on performance assessment of nuclear waste repositories included talks by P. Swift (SNL) and R.C. Ewing (Univ. of Michigan). In the session on ceram-

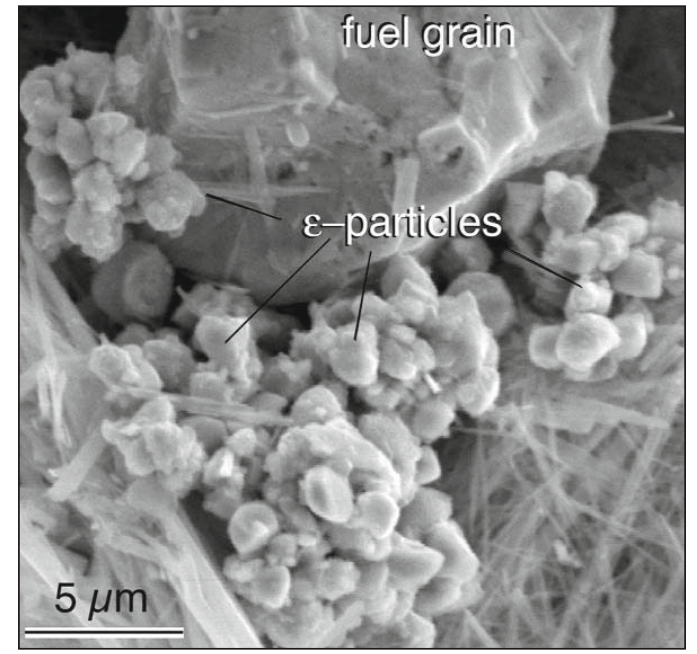

Figure 1. Scanning electron micrograph of corroded spent nuclear fuel showing Mo- and Tcrich $\varepsilon$ particles concentrated on the surface of a corroded $\mathrm{UO}_{2}$ fuel grain. In the foreground are acicular uranium (VI) silicates that formed during the corrosion process. ic waste forms, B.E. Burakov (Khlopin Radium Inst., Russia) presented results of a study of self-irradiation of plutonium phosphate and lanthanium phosphate doped with ${ }^{238} \mathrm{Pu}$.

P.V. Iseghem (SCK CEN Mol, Belgium) opened a session on glass waste forms with a presentation on the status of the European GLAMOR project, a consortium effort to understand long-term dissolution rates of high-level nuclear waste glasses. In a session on waste/tank, site cleanup, and decommissioning, G.W. Koroll (AECL, Canada) discussed the programmatic, technical, and practical challenges of decommissioning a nuclear site (Whiteshell Laboratories, Manitoba, Canada).

Symposium Support: Southwest Research Inst. 


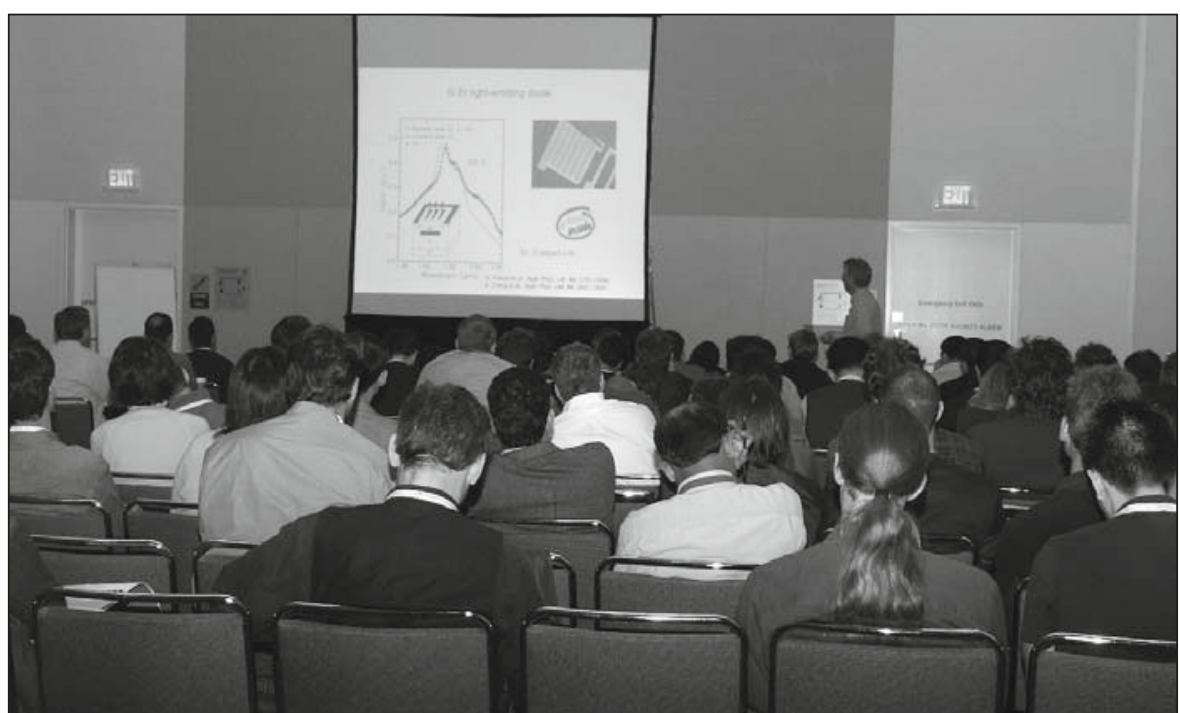

MRS tutorials concentrate on rapidly breaking areas of research and provide overviews of established fields.

Building on the NSF GK-12 program, several faculty, staff, and graduate students D. Hammer (Univ. of Maryland) and F.M. Goodchild (UCSB) demonstrated new approaches for engaging graduate student fellows in the K-12 classroom. These innovative programs provide a different type of teaching assistant experience while at the same time cultivating future scientists and engineers. L. Bartolo (Kent State) and others described current and future efforts with the Materials Digital Library and the NSF National Digital Science Library Program. Several speakers exhibited new methods for introducing computation into the materials science and engineering classroom. S. Whittingham (SUNY, Binghamton) and others highlighted a series of projects involved with incorporating nanotechnology into the undergraduate curriculum. These efforts emphasized the important, interdisciplinary role of materials science and engineering in bringing novel technologies and research into the undergraduate classrooms in chemistry, physics, biology, and engineering. The symposia concluded with a dynamic and interactive panel discussion on "Materials Education: What's the Problem?" This discussion was motivated by the article by Randy Bass titled "The Scholarship of Teaching: What's the Problem?" which can be found at http:/ / www.doit.gmu. edu/Archives/feb98/randybass.htm. The published proceedings of the symposium will appear as a special issue of the Journal of Materials Education in the Fall of 2004.

Symposium Support: Univ. of Maryland.

\begin{tabular}{|llll|}
\hline & \multicolumn{1}{c|}{ MRS Future Meetings } \\
\hline 2004 FALL MEETING & 2005 SPRING MEETING & 2005 FALL MEETING & 2006 SPRING MEETING \\
Nov. 29-Dec. 3 & March 28-April 1 & Nov. 28-Dec. 2 & April 17-21 \\
Exhibit: Nov. 30-Dec. 2 & Exhibit: March 29-31 & Exhibit: Nov. 29-Dec. 1 & Exhibit: April 18-20 \\
Boston, MA & San Francisco, CA & Boston, MA & San Francisco, CA \\
\hline
\end{tabular}

